Review

\title{
A Systematic Review of the Biological Processes Involved in Deep-Brain Stimulation for Parkinson's disease: A Focus on the Potential Disease-Modifying Effects
}

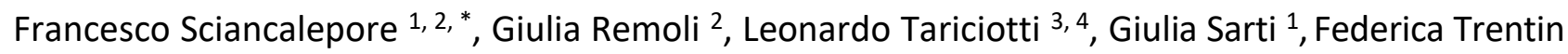
${ }^{5}$, Gabriele Riccardi ${ }^{6}$, Eleonora Lacorte ${ }^{2}$, Massimo Corbo ${ }^{7}$, Marco Canevelli ${ }^{1,2}$, Nicola Vanacore ${ }^{2}$, Paola Piscopo ${ }^{8}$

1. Department of Human Neuroscience, "Sapienza" University of Rome, Rome, Italy; E-Mails: sciancalepore.francesco19@gmail.com; giusarti95@gmail.com; marco.canevelli@iss.it

2. National Center for Disease Prevention and Health Promotion, Italian National Institute of Health, Viale Regina Elena, 299, 00161 Rome, Italy; E-Mails: giulia.remoli@guest.iss.it; eleonora.lacorte@iss.it; nicola.vanacore@iss.it

3. Unit of Neurosurgery, IRCSS Fondazione Cà Grande Ospedale Maggiore Policlinico, University of Milan, Via Francesco Sforza, 35, 20122 Milan, Italy; E-Mail: leonardotariciottimd@gmail.com

4. Department of Oncology and Hemato-oncology, University of Milan, Via Festa del Perdono 7, 20122 Milan, Italy

5. Department of Oncological and Degenerative Spine Surgery, IRCCS, Rizzoli Orthopedic Institute, Via G.C. Pupilli, 1, 40136 Bologna, Italy; E-Mail: federicatrentin94@gmail.com

6. Department of Sense Organ, Sapienza University of Rome, Viale del Policlinico, 155, 00161 Rome, Italy; E-Mail: ric.gabriele@gmail.com

7. Department of Neurorehabilitation Sciences, Casa Cura Policlinico, Via Dezza 48, 20144 Milan, Italy; E-Mail: m.corbo@ccppdezza.it

8. Department of Neuroscience, Italian National Institute of Health, Viale Regina Elena, 299, 00161 Rome, Italy; E-Mail: paola.piscopo@iss.it

* Correspondence: Francesco Sciancalepore; E-Mail: sciancalepore.francesco19@gmail.com

Academic Editor: Lilach Soreq

Special Issue: Deep Brain Stimulation for Neurobiology Diseases

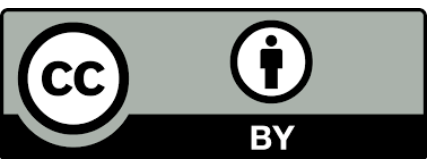

(C) 2021 by the author. This is an open access article distributed under the conditions of the Creative Commons by Attribution License, which permits unrestricted use, distribution, and reproduction in any medium or format, provided the original work is correctly cited. 
OBM Neurobiology

2021, volume 5 , issue 2

doi:10.21926/obm.neurobiol.2102097
Received: February 05, 2021

Accepted: May 02, 2021

Published: May 19, 2021

\begin{abstract}
Deep-Brain Stimulation (DBS) is an important treatment option for the management of Parkinson's disease (PD) and is a common symptomatic treatment. However, an increasing number of studies have examined the biological processes to assess if DBS can also modify the natural history of PD by acting on its pathophysiological mechanisms. Relevant literature published up to November 2020 was systematically searched on databases such as PubMed, ISI Web of Knowledge, Academic Search Index, and Science Citation Index. The following predefined inclusion criteria were applied to the full-text versions of the selected articles: i) recruiting and monitoring of PD subjects that were previously treated with DBS and ii) investigating the electrophysiological, biochemical, epigenetic, or neuroimaging effects of DBS. Studies focusing exclusively on motor and clinical changes were excluded. Reviews, case reports, studies on animal models, and computational studies were also not considered. Out of 2,960 records screened, 43 studies met the inclusion criteria. Only three studies described a potential disease-modifying effect of DBS. However, a wide heterogeneity was observed in the investigated biomarkers, and the design and methodological issues of several studies limited their ability to find potential disease-modifying features. Specifically, $60.4 \%$ of the trials followed-up subjects for no more than 1 year from the surgical intervention, and $67.4 \%$ observed patients with PD only once after DBS. Moreover, $64.2 \%$ of the studies enrolled late-stage PD patients. Most of the studies $(88.4 \%)$ reported that DBS only had a symptomatic effect, with several of them showing some limitations in the study design and recruitment of patients. Further studies using shared biomarkers are encouraged to assess if and how DBS might affect the progression of PD. Based on the existing preclinical literature, prospective clinical trials examining the course of PD in early-stage patients are needed.
\end{abstract}

\title{
Keywords
}

Deep brain stimulation (DBS); Parkinson's disease (PD); disease-modifying; systematic review; biomarkers

\section{Introduction}

Parkinson's disease (PD) is the second-most common neurodegenerative disorder with a prevalence of $2-3 \%$ in individuals aged $\geq 65$ years [1]. PD is characterized by motor signs such as bradykinesia, resting tremor, muscular rigidity, and postural disturbances. Its clinical manifestations also include a broad spectrum of non-motor symptoms, including cognitive changes, mood disorders, and autonomic dysfunctions. The main underlying neuropathological hallmarks of PD are the intracellular accumulation of misfolded $\alpha$-synuclein, and the progressive degeneration of dopaminergic neurons in the substantia nigra pars compacta (SNpc) $[1,2]$. 
There are currently no available disease-modifying treatments for PD. The main intervention to manage motor symptoms is dopamine replacement through L-Dopa. Dopamine agonists, catechol-O-methyl transferase inhibitors, monoamine oxidase-B (MAO-B) inhibitors, and non-dopaminergic drugs (i.e., anticholinergics and amantadine) are also used as symptomatic agents [3].

Another treatment option is Deep Brain Stimulation (DBS), which is considered to be a useful strategy to manage motor symptoms. DBS is a surgical procedure that involves the delivery of continuous electrical stimulation to a given neural target through chronically implanted electrodes [4]. DBS has been associated with some relevant side effects such as behavioral changes, depression, and cognitive impairment [5-8]. However, most studies have found it to be safe and well-tolerated [9]. Electrodes are often inserted in the Subthalamic Nucleus (STN) or the internal Globus Pallidus (GPi), as several clinical trials have established that the stimulation of these two areas is often associated with an improvement of motor symptoms in patients with PD $[9,10]$.

To date, the exact mechanisms underlying the effects of this neurosurgical procedure have only been partially elucidated. The main current theory on the underlying mechanism of DBS is that it induces changes in the firing pattern of the basal ganglia structures and pathways by inhibiting or exciting neuronal activity in the STN or GPi. For example, the neuronal activity in the GPi was observed to increase after DBS in the STN [11]. Another theory called "the disruption hypothesis" has proposed that DBS dissociates both input and output information and blocks unusual signals through the cortico-basal-ganglia loop [12]. Overall, these hypotheses suggest that the effects induced by DBS are only transient, supporting the common opinion within the neurological community that DBS, like pharmacological therapies, is not a disease-modifying treatment for PD $[12,13]$. Accordingly, some studies have reported DBS to be a symptomatic treatment with limited long-term improvements $[14,15]$. However, increasing studies have focused on investigating the biological and neurophysiological correlations of DBS to find a potential disease-modifying effect on the progression of PD. Accordingly, some preclinical studies have suggested the association of STN-DBS with an improvement of dopaminergic neuron survival and an increase in the levels of Brain-Derived Neurotrophic Factor (BDNF), suggesting a long-term neuroprotective effect $[16,17]$. This hypothesis could also be associated with the functional inhibition of STN by DBS, which reduces the toxicity of nigral glutamate. However, it is not supported in humans, probably due to the use of DBS in patients with late-stage PD $[18,19]$.

Therefore, this study aimed to systematically review and discuss the existing evidence on the biological effects associated with DBS treatment in patients with PD to explore the potential disease-modifying features of this treatment. The objective of this study was to understand if DBS could be considered only as a symptomatic treatment for PD or if it may induce modifications that might affect the natural history of PD by acting on its pathophysiological mechanisms.

\section{Methods}

This systematic literature review was performed according to the methodology described in the Cochrane handbook for systematic reviews [20] and was reported based on the PRISMA statement for reporting systematic reviews and meta-analyses [21]. All the literature published up to November 2020 was retrieved by a thorough search of the databases "PubMed", "ISI Web of Knowledge", and "Discovery" using the search terms parkinson* AND ("deep brain stimulation" 
OR "deep brain stimulations" OR "DBS") AND (biol* OR chemi* OR biochemi* OR bio-chemi* OR neurobiol* OR neuro-biol* OR neurochem* OR neuro-chemi* OR biomark* OR neurophysiol* OR neuro-physiol* OR "disease modifying" OR "disease modify" OR disease-modifying OR disease-modify OR "disease modification" OR neuroprotect*).

No limitations in the search strategy were applied to the date of publication, study design, or language. References of the selected studies were also searched to identify any further relevant data.

The title and abstracts of the identified records were initially screened and selected by six independent reviewers (FS, GR, PP, GS, FT, and GR) based on their pertinence to the review topic. Disagreements were resolved by a consensus.

The following set of predefined inclusion criteria were then individually applied to the selected articles in their full-text version: i) recruiting and monitoring of PD subjects that were previously treated with DBS and ii) investigating the electrophysiological, biochemical, epigenetic, or neuroimaging effects of DBS. Studies with an exclusive focus on motor and clinical changes were excluded. Reviews, case reports, studies on animal models, and computational studies were also not considered. Articles not published in English were removed. Systematic reviews were considered separately to check the consistency of data.

Data were extracted by three pairs of independent reviewers (GR and LT, PP and GS, FT and GR) using specifically designed tables. The main clinical and demographic features of all included studies were first reported in an introductory table (Table 1), while the analytical procedures and results from the biochemical and epigenetic studies (Table 2a) and neurophysiological studies (Table $2 b$ ) were reported in another table. 
Table 1 Clinical and demographic data of the included studies according to the type.

\begin{tabular}{|c|c|c|c|c|c|c|c|c|}
\hline Study & $\begin{array}{l}\text { Diagnostic } \\
\text { criteria }\end{array}$ & $\begin{array}{l}\text { Surgery } \\
\text { selection criteria }\end{array}$ & DBS & $\begin{array}{l}\text { Pharmacologi } \\
\text { cal interventions }\end{array}$ & $\begin{array}{l}\text { Follow-up } \\
\text { (When) }\end{array}$ & $\begin{array}{l}\text { Participants } \\
\text { (number; me } \\
\text { female/male) } \\
\text { Cases }\end{array}$ & $\begin{array}{l}\text { an age; } \\
\text { Controls }\end{array}$ & $\begin{array}{l}\text { UPDRS pre } \\
\text { and } \\
\text { post-surgery } \\
\text { (cases) }\end{array}$ \\
\hline \multicolumn{9}{|l|}{ BIOCHEMICAL } \\
\hline $\begin{array}{l}\text { Constantinescu } \\
2011 \\
{[22]}\end{array}$ & $\begin{array}{l}\text { PD } \\
\text { (UK Parkinson's } \\
\text { Disease Brain Bank } \\
\text { criteria) }\end{array}$ & NR & $\begin{array}{l}\text { STN } \\
\text { Bilat. }\end{array}$ & NR & $\begin{array}{l}1 \text { pre-DBS } \\
4 \text { post-DBS } \\
\text { (1 week; } \\
2 \text { weeks; } \\
4.5 \text { months; } \\
1 \text { year) }\end{array}$ & $\begin{array}{l}8 ; \\
58.5 \\
(51-63) ; \\
2 / 6\end{array}$ & - & NR \\
\hline $\begin{array}{l}\text { Constantinescu } \\
2018 \\
{[23]}\end{array}$ & $\begin{array}{l}\text { PD } \\
\text { (Not specified } \\
\text { criteria) }\end{array}$ & NR & $\begin{array}{l}\text { STN } \\
\text { Bilat }\end{array}$ & $\begin{array}{l}\text { L-DOPA, } \\
\text { apomorphine, } \\
\text { COMT-inhibitors, } \\
\text { MAO-B } \\
\text { inhibitors, } \\
\text { and } \\
\text { amantadine }\end{array}$ & $\begin{array}{l}>1 \text { pre-DBS } \\
\text { (during } 1 \\
\text { year) } \\
\geq 2 \text { post-DBS } \\
\text { (during } 11 \\
\text { years) }\end{array}$ & $\begin{array}{l}16 \\
64(55-75) \\
6 / 10\end{array}$ & - & NR \\
\hline $\begin{array}{l}\text { Dong } \\
2019 \\
{[24]}\end{array}$ & $\begin{array}{l}\text { PD } \\
\text { (Movement } \\
\text { Disorder Society } \\
\text { criteria) }\end{array}$ & NR & NR & NR & $\begin{array}{l}1 \text { post-DBS } \\
\text { (1 month) }\end{array}$ & $\begin{array}{l}4 \text { PD1 (NO } \\
\text { DBS) } \\
4 \text { PD2 (DBS } \\
\text { OFF) } \\
4 \text { PD3 (DBS } \\
\text { ON); } \\
72(65-79) ; \\
4 / 8\end{array}$ & $\begin{array}{l}12 ; \\
69(65-71) ; \\
3 / 9\end{array}$ & NR \\
\hline
\end{tabular}




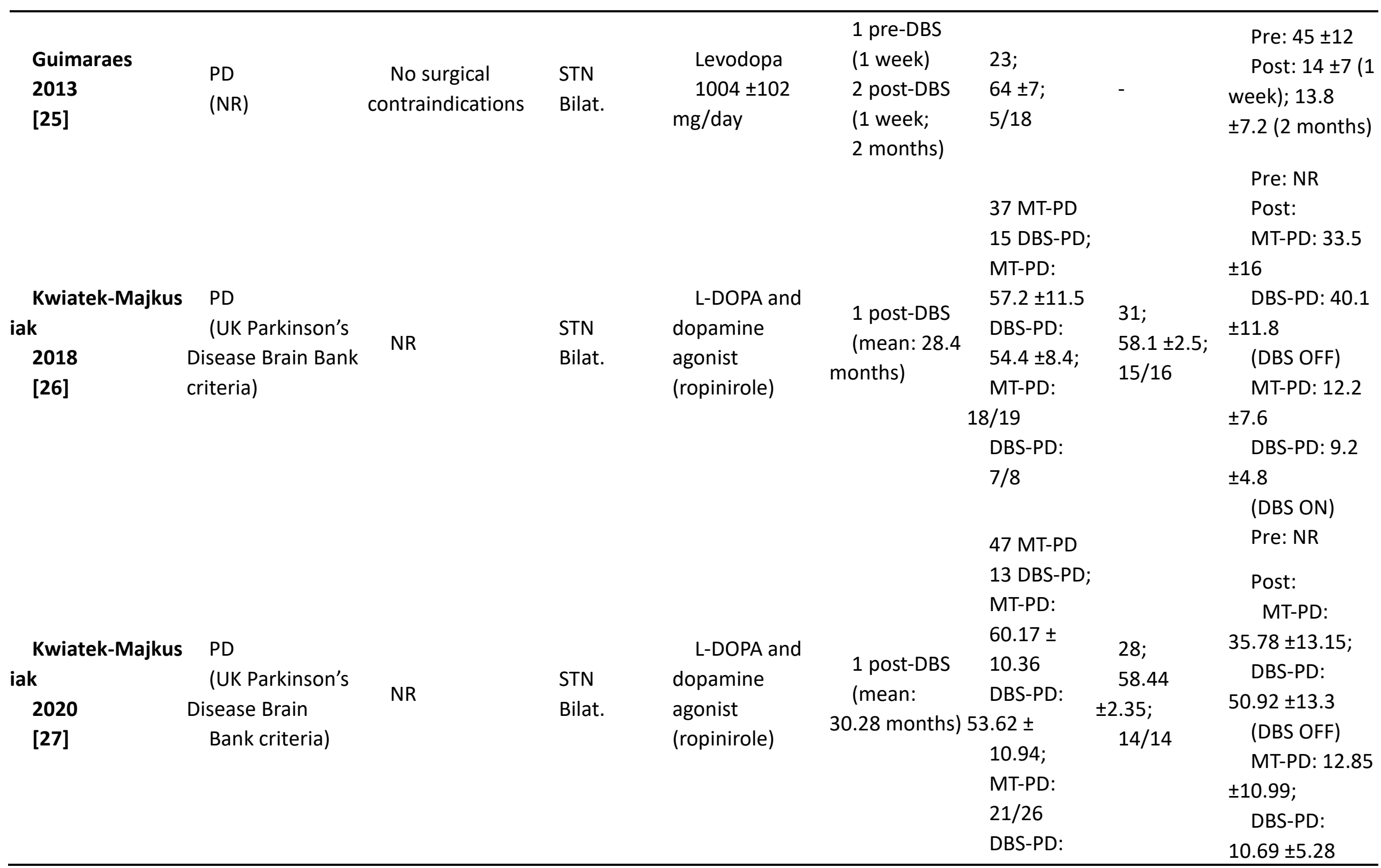




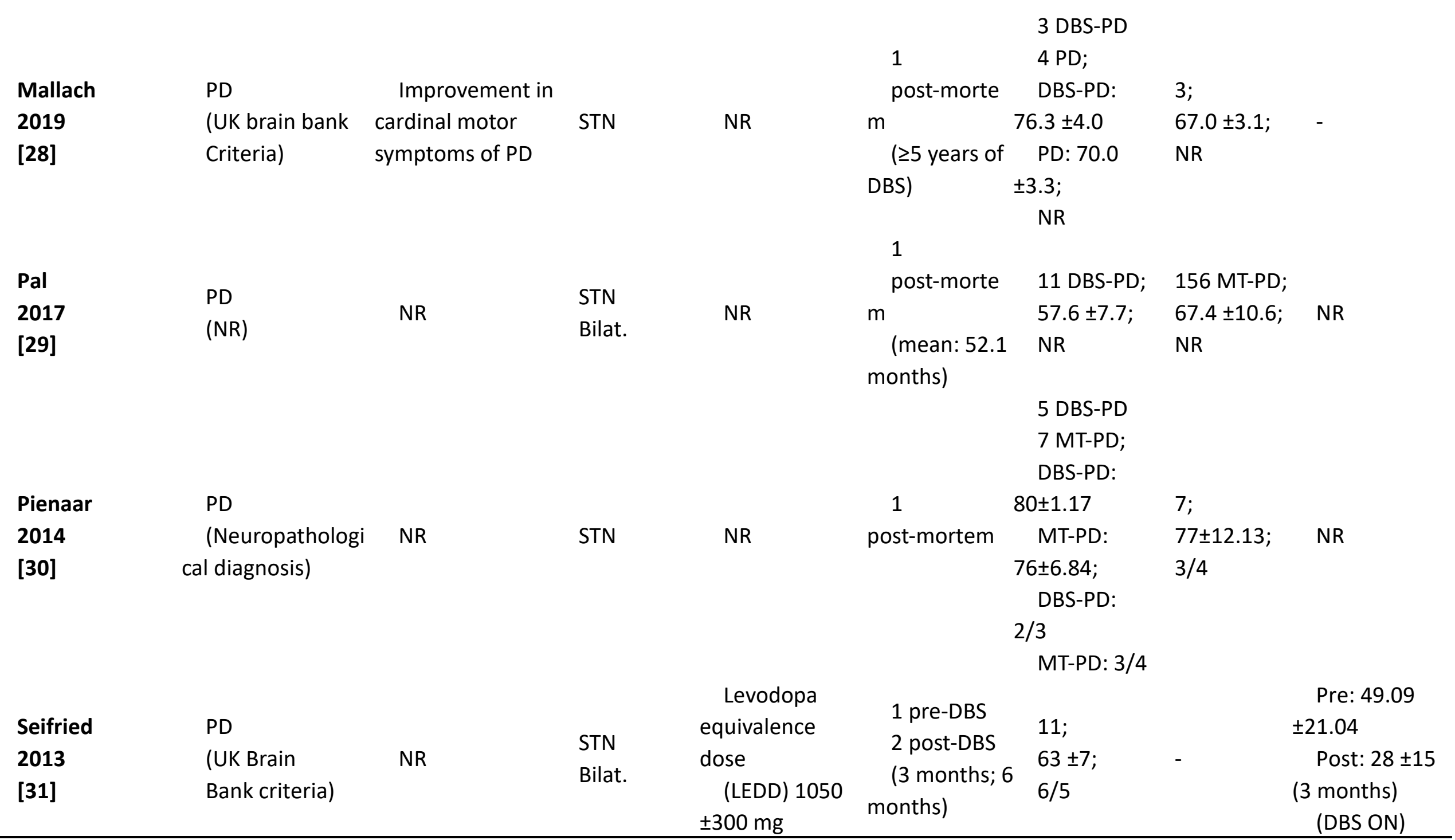




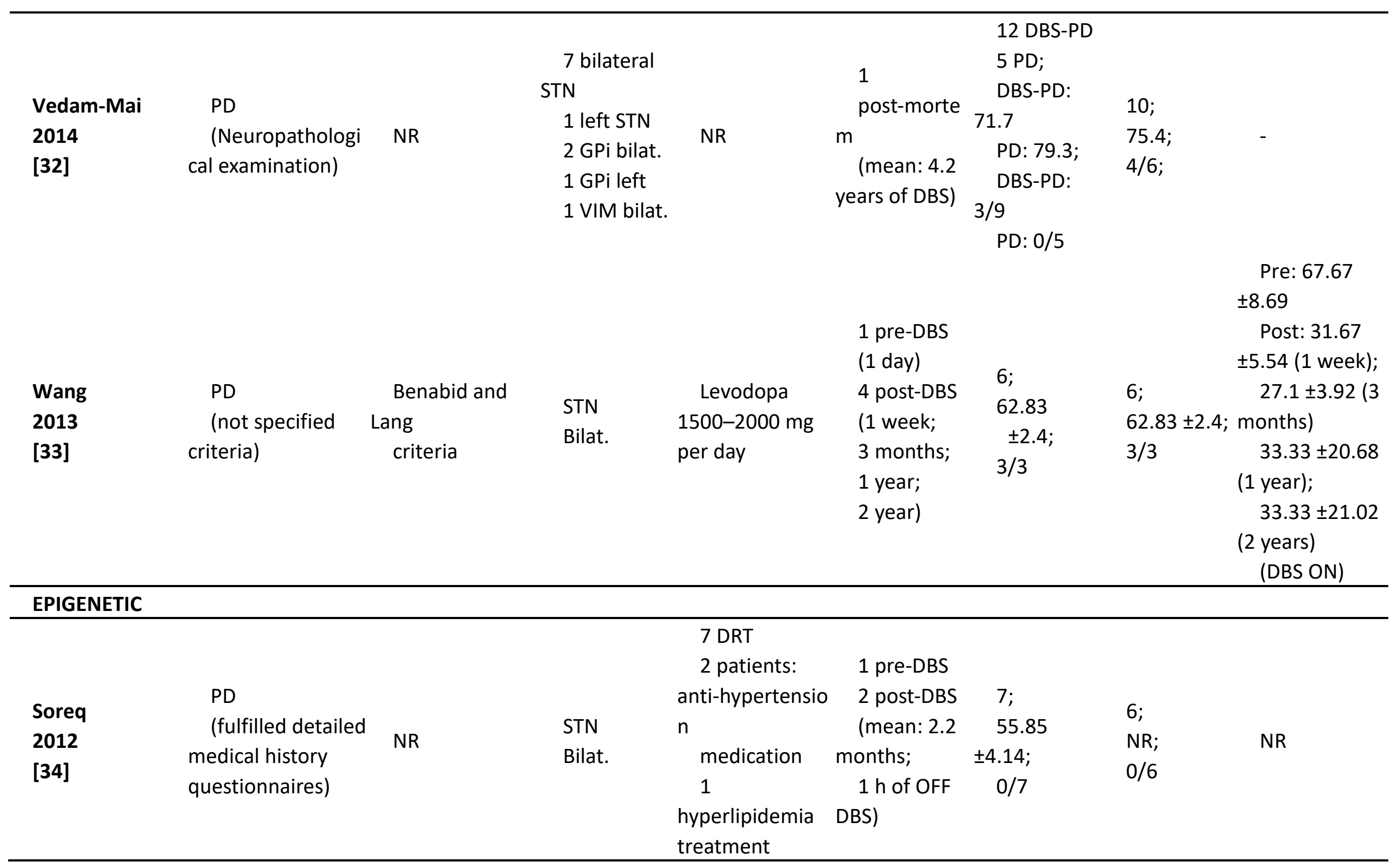




\begin{tabular}{|c|c|c|c|c|c|c|c|c|}
\hline $\begin{array}{l}\text { Soreq } \\
2013 a \\
{[35]}\end{array}$ & See above & See above & See above & See above & See above & See above & See above & See above \\
\hline $\begin{array}{l}\text { Soreq } \\
2013 b \\
{[36]}\end{array}$ & See above & See above & See above & See above & See above & See above & See above & See above \\
\hline $\begin{array}{l}\text { Soreq } \\
2014 \\
{[37]}\end{array}$ & See above & See above & $\begin{array}{l}\text { STN } \\
\text { Bilat. }\end{array}$ & NR & See above & $\begin{array}{l}3 ; \\
52.7 \\
0 / 3\end{array}$ & $\begin{array}{l}3 \\
60.7 \\
0 / 3\end{array}$ & $\begin{array}{l}\text { Pre: NR } \\
\text { Post: } 34 \text { (DBS } \\
\text { ON) } \\
\quad 42.5 \text { (DBS } \\
\text { OFF) }\end{array}$ \\
\hline ELECTROPH & LOGICAL & & & & & & & \\
\hline $\begin{array}{l}\text { Airaksinen } \\
2012 \\
{[38]}\end{array}$ & $\begin{array}{l}P D \\
(N R)\end{array}$ & NR & $\begin{array}{l}\text { STN } \\
\text { Bilat. }\end{array}$ & $\begin{array}{l}\text { Optimized } \\
\text { antiparkinsonian } \\
\text { medical therapy }\end{array}$ & $\begin{array}{l}1 \text { post-DBS } \\
\text { (mean: } 1.02 \\
\text { years) }\end{array}$ & $\begin{array}{l}11 ; \\
61.4 \pm 6.7 \\
6 / 5\end{array}$ & - & $\begin{array}{l}\text { Pre: NR } \\
\text { Post: } 27.7 \\
\pm 12.9\end{array}$ \\
\hline $\begin{array}{l}\text { Anidi } \\
2018 \\
{[39]}\end{array}$ & $\begin{array}{l}\text { Patients with } \\
\text { history or } \\
\text { presenting freezing } \\
\text { of gait (FOG) during } \\
\text { tasks were defined } \\
\text { "Freezers". Patients } \\
\text { not presenting FOG } \\
\text { were defined } \\
\text { "Non-Freezers" }\end{array}$ & NR & $\begin{array}{l}\text { STN } \\
\text { Bilat. }\end{array}$ & $\begin{array}{l}\text { Long-acting } \\
\text { dopaminergic } \\
\text { medications }\end{array}$ & $\begin{array}{l}1 \text { post-DBS } \\
\text { ( } \geq 21 \\
\text { months) }\end{array}$ & $\begin{array}{l}9 \\
\text { Freezers; } \\
62.21 \\
\pm 7.10 \\
4 / 5\end{array}$ & $\begin{array}{l}4 \\
\text { Non } \\
\text { Freezers; } \\
62.37 \\
\pm 8.12 ; \\
\quad \text { NR }\end{array}$ & $\begin{array}{l}\text { Pre: } \\
45.17 \pm 7.83 \\
\text { (Meds OFF) } \\
19.57 \pm 8.66 \\
\text { (Meds ON) } \\
\text { Post: } \\
36.38 \pm 10.20 \\
\text { (DBS OFF) } \\
12.63 \pm 7.21 \\
\text { (DBS ON) }\end{array}$ \\
\hline $\begin{array}{l}\text { Dauper } \\
2002 \\
{[40]}\end{array}$ & $\begin{array}{l}\text { Akinetic-rigid PD } \\
\text { (NR) }\end{array}$ & NR & $\begin{array}{l}\text { STN } \\
\text { Bilat. }\end{array}$ & $\begin{array}{l}\text { Optimized } \\
\text { antiparkinsonian } \\
\text { medical therapy }\end{array}$ & $\begin{array}{l}1 \text { post-DBS } \\
\text { ( } \geq 3 \text { months) }\end{array}$ & $\begin{array}{l}8 \\
59.3 \pm 10.0 \\
4 / 4\end{array}$ & $\begin{array}{l}10 \\
N R \\
6 / 4\end{array}$ & $\begin{array}{l}\text { Pre: } 10.8 \pm 7.1 \\
\text { (1 PD patient) } \\
\text { Post: }\end{array}$ \\
\hline
\end{tabular}




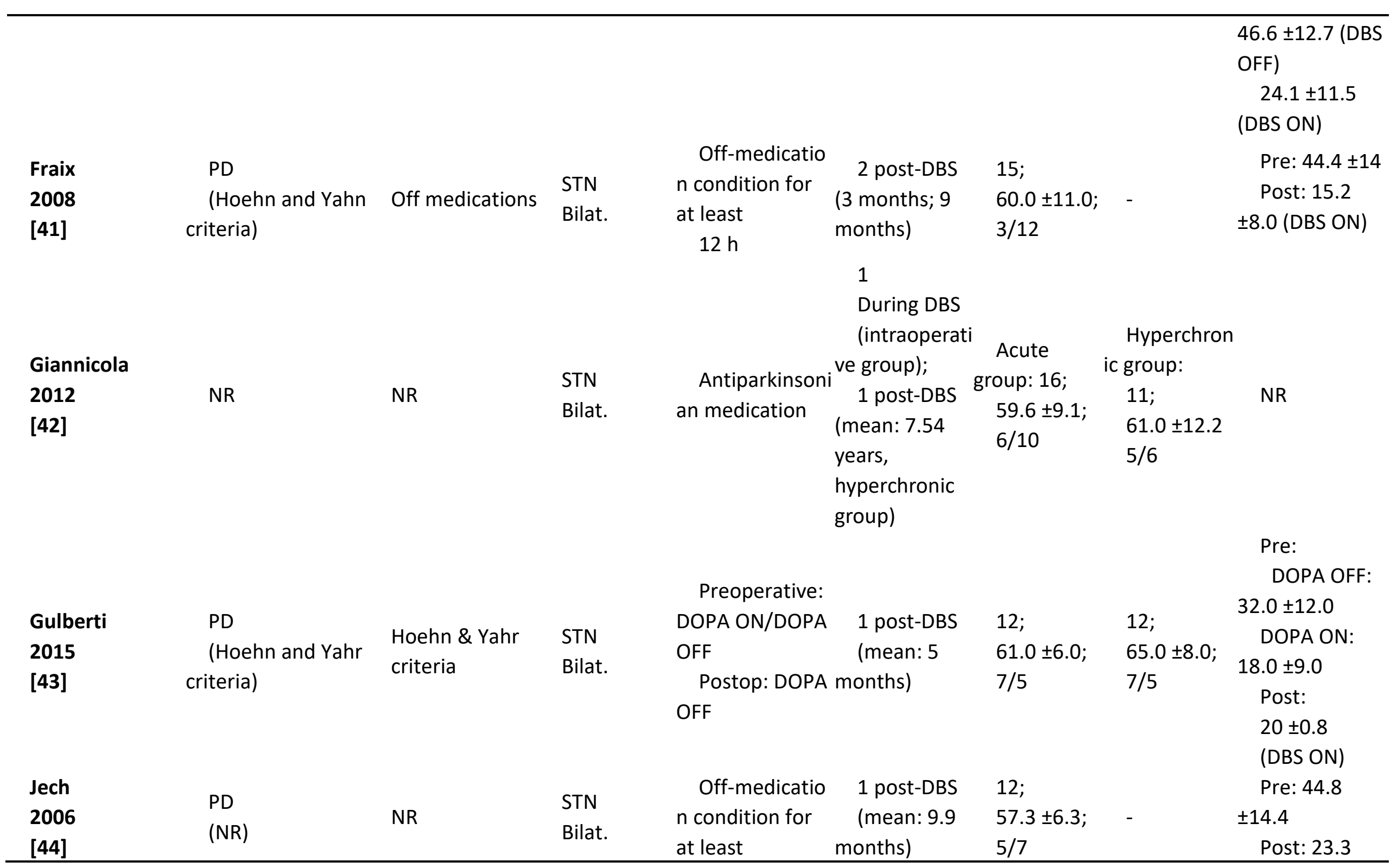


$12 \mathrm{~h}$

$\pm 12.0$

(DBS ON)

Pre: 51.1

\begin{tabular}{|c|c|c|c|c|c|c|}
\hline $\begin{array}{l}\text { Michmizos } \\
2015 \\
{[45]}\end{array}$ & $\begin{array}{l}\text { PD } \\
\text { (Hoehn and Yahn } \\
\text { criteria) }\end{array}$ & $\begin{array}{l}\text { CAPSIT } \\
\text { criteria }\end{array}$ & $\begin{array}{l}\text { STN } \\
\text { Bilat. }\end{array}$ & NR & $\begin{array}{l}1 \text { post-DBS } \\
\text { ( } \geq 2 \text { years) }\end{array}$ & $\begin{array}{l}9 ; \\
\text { NR; } \\
\text { NR }\end{array}$ \\
\hline
\end{tabular}

Dopaminergic

\begin{tabular}{|c|c|c|c|c|c|}
\hline Pierantozzi & PD & Hoeh & $\begin{array}{l}4 \text { bilateral } \\
\text { GPi-DBS }\end{array}$ & $\begin{array}{l}\text { therapy } \\
\text { before }\end{array}$ & 1 post-DBS \\
\hline $\begin{array}{l}1999 \\
{[46]}\end{array}$ & $\begin{array}{l}\text { (Hoehn and Yahr } \\
\text { criteria) }\end{array}$ & criteria & $\begin{array}{l}2 \text { bilateral } \\
\text { STN-DBS }\end{array}$ & $\begin{array}{c}\text { and after } 3 \mathrm{~h} \\
\text { of apomorphine }\end{array}$ & $\begin{array}{l}\text { (mean: } 6 \\
\text { months) }\end{array}$ \\
\hline
\end{tabular}

$6 ;$ 51.6 NR

Post: $26.7 \pm 7$

.7 (DBS ON)

Pre: 73.9

$\pm 10.2$

Post:

GPi-DBS: 17.0

$\pm 7.7$

(DBS ON);

STN-DBS:

$20.0 \pm 1.4$

(DBS ON)

Pre: NR

Post:

The off drugs

motor

UPDRS scores

$\downarrow 41 \%(\mathrm{p}=$

0,01); the

bradykinesia/rig

idity UPDRS

scores $\downarrow 37 \%$

( $p=0,01)$ the

tremor 
$\downarrow 59 \%(p=$

$0.05)$

LIMPE:

Rosa

2011

[48]

(NR)

Guidelines for the treatment of

Parkinson's

Disease

$\begin{array}{ll}\text { Sinclair } & \text { PD } \\ 2018 & \text { (NR) }\end{array}$

[49]

(NR)

NR

\section{Clinical motor}

outcome of

bilateral

subthalamic

Akinetic rigid nucleus

(AR) or Tremor deep-brain

Trager

2016 dominant (TD),

stimulation for

using the following Parkinson's

criteria (Quinn et disease using
al., 2015)
image-guided

frameless

stereotaxy

(Bronte-Stewar

$t$ et al. 2010)

$\begin{array}{llll}\text { Weiss } & \text { PD } & \text { Hoehn and Yahr } & \text { STN } \\ \mathbf{2 0 1 5} & \text { (NR) } & \text { criteria } & \text { Bilat. }\end{array}$

\section{STN \\ Bilat.}

STN

Bilat.

$$
\begin{aligned}
& \text { Antiparkinsoni } \\
& \text { an } \\
& \text { medication } \\
& \text { (Levodopa) }
\end{aligned}
$$

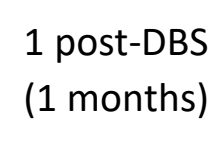

$7 ;$

\section{1}

Levodopa

post-DBS

( $\geq 3$ months) $5 / 9$

Long-acting

dopaminergic

medications

were withdrawn

over $24 \mathrm{~h}$

and

Bilat.

short-acting

medication was

withdrawn

over $12 \mathrm{~h}$

before surgery

\section{1 pre-DBS \\ 1 post-DBS}

OFF

(1 month)

2 post-DBS

ON

(6 months;

1 year)

Levodopa

1 post-DBS

(mean: 2.9
$66.8 \pm 5.4 ;$

$1 / 6$

14 ;

17 ;

$61.6 \pm 8.04$; -

$5 / 12$

Pre:

DOPA ON:

$19.7 \pm 5.0$

DOPA OFF:

$37.7 \pm 4.3$

Post: NR

NR

Pre: 42.5

$\pm 10.6$

Post: $\downarrow$ score

$p=0.04$

(12 months)

Pre: 57.0

$58.6 \pm 9.4$. 


\begin{tabular}{|c|c|c|c|c|c|c|c|c|}
\hline [51] & & & & & jears) & $5 / 15$ & & $\begin{array}{l}\text { Post: } 22.3 \\
\pm 9.7 \\
\text { (DBS ON) } \\
\end{array}$ \\
\hline \multicolumn{9}{|c|}{ NEUROIMAGING } \\
\hline $\begin{array}{l}\text { Dong } \\
2020 \\
{[52]}\end{array}$ & $\begin{array}{l}\text { "Definite } \\
\text { diagnosis of } \\
\text { idiopathic PD" } \\
\text { (NR criteria) }\end{array}$ & NR & $\begin{array}{l}\text { STN } \\
\text { Bilat. }\end{array}$ & $\begin{array}{l}\text { Compound } \\
\text { levodopa and } \\
\text { dopamine } \\
\text { receptor } \\
\text { agonists }\end{array}$ & $\begin{array}{l}1 \text { pre-DBS } \\
1 \text { post-DBS } \\
\text { ( } 3 \text { months) }\end{array}$ & $\begin{array}{c}23 \\
60.91 \\
\pm 12.62 \\
14 / 9\end{array}$ & $\begin{array}{c}14 ; \\
63.29 \\
\pm 9.72 \\
7 / 7\end{array}$ & $\begin{array}{l}\text { Pre: } 39.30 \\
\pm 12.47 \\
\text { Post: NR }\end{array}$ \\
\hline \multirow{11}{*}{$\begin{array}{l}\text { Ge } \\
2020 \\
{[53]}\end{array}$} & & \multirow{11}{*}{ NR } & \multirow{11}{*}{$\begin{array}{l}\text { PD2: STN } \\
\text { Bilat. }\end{array}$} & \multirow{11}{*}{$\begin{array}{l}\text { Oral } \\
\text { antiparkinsonian } \\
\text { treatment }\end{array}$} & \multirow{11}{*}{$\begin{array}{l}1 \text { pre-DBS } \\
2 \text { post-DBS } \\
\text { ( } 3 \text { months: } \\
1 \text { year) }\end{array}$} & \multicolumn{3}{|r|}{$\begin{array}{l}\text { Pre: } \\
\text { PD1: } 25.2 \\
\pm 14.4\end{array}$} \\
\hline & & & & & & PD1:33 & HC1: 33 & PD2: 49.7 \\
\hline & & & & & & $\begin{array}{l}\text { PD2: 9; } \\
\text { PD1. 58 } 1\end{array}$ & $\begin{array}{l}\text { HC2: 9; } \\
\text { HC1. }\end{array}$ & \pm 8.4 \\
\hline & (UK Parkinson's & & & & & $\begin{array}{l}\text { PD1: } 58.1 \\
\pm 10.3\end{array}$ & $\begin{array}{l}\text { HCI: } \\
57.4 \pm 10.5\end{array}$ & $\begin{array}{l}\text { Post: } \\
\text { PD2: } 27.4\end{array}$ \\
\hline & Disease Brain Bank & & & & & PD2: 63.1 & $\mathrm{HC2:} 61.7$ & \pm 17.3 \\
\hline & criteria) & & & & & \pm 9.2 & \pm 7.3 & (3 months; \\
\hline & & & & & & PD1:18/15 & HC1: 18/15 & DBS OFF) \\
\hline & & & & & & PD2:5/4 & $\mathrm{HC2}: 5 / 4$ & PD2: 49.3 \\
\hline & & & & & & & & \pm 18.2 \\
\hline & & & & & & & & (1 year; DBS \\
\hline & & & & & & & & OFF) \\
\hline $\begin{array}{l}\text { Hanssen } \\
2019 \\
{[54]}\end{array}$ & $\begin{array}{l}\text { PD } \\
\text { (Movement } \\
\text { Disorder Society } \\
\text { criteria) }\end{array}$ & NR & $\begin{array}{l}\text { STN } \\
\text { Bilat. }\end{array}$ & $\begin{array}{l}\text { Levodopa } \\
\text { equivalence } \\
\text { dose of } 552 \pm 351 \text { y } \\
\mathrm{mg} / \text { day }\end{array}$ & $\begin{array}{l}1 \text { post-DBS } \\
\text { (mean: } 2.2 \\
\text { jears) }\end{array}$ & $\begin{array}{l}26 \\
N R \\
N R\end{array}$ & - & $\begin{array}{l}\text { Pre: NR } \\
\text { Post: } \\
\text { 个 } 26.4 \\
\pm 15.5 \% \text { of the } \\
\text { UPDRS-III }\end{array}$ \\
\hline
\end{tabular}




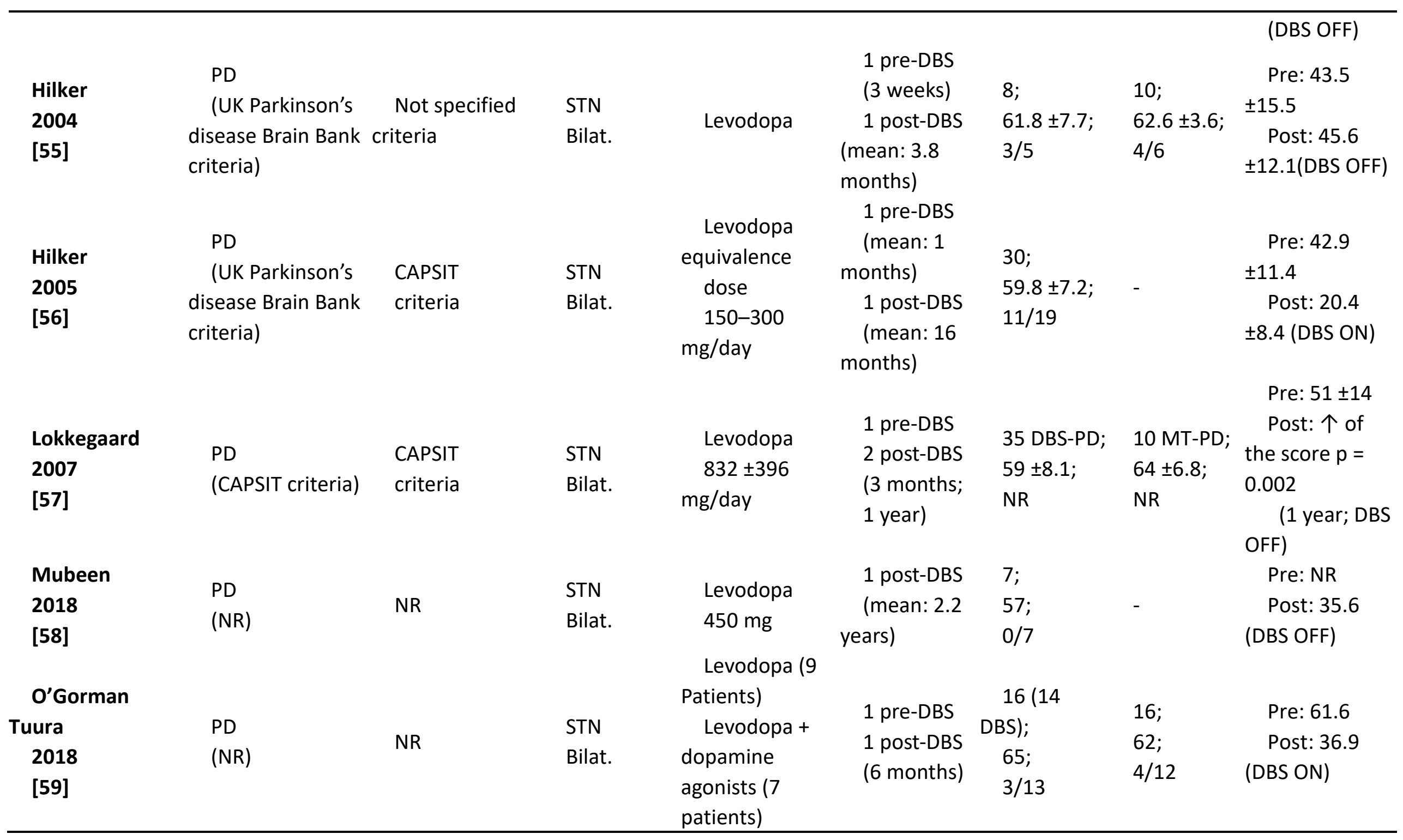




\begin{tabular}{|c|c|c|c|c|c|c|c|c|}
\hline $\begin{array}{l}\text { Palard-Novello } \\
2020 \\
{[60]}\end{array}$ & $\begin{array}{l}\text { PD } \\
\text { (UK Parkinson's } \\
\text { disease Brain Bank } \\
\text { criteria) }\end{array}$ & $\begin{array}{l}\text { Australian } \\
\text { guidelines } \\
\text { (Movement } \\
\text { Disorder Society } \\
\text { of Australia) }\end{array}$ & $\begin{array}{l}\text { GPi } \\
\text { Bilat. }\end{array}$ & $\begin{array}{l}\text { Levodopa } \\
\text { equivalent daily } \\
\text { dose } \\
1446 \pm 627 \mathrm{mg}\end{array}$ & $\begin{array}{l}1 \text { pre-DBS } \\
\text { (4 months) } \\
1 \text { post-DBS } \\
\text { (4 months) }\end{array}$ & $\begin{array}{l}32 \\
60.9 \pm 7.7 \\
17 / 15\end{array}$ & - & $\begin{array}{l}\text { Pre: } 39 \pm 15 \\
\text { Post: } 26 \pm 13 \\
\text { (DBS ON) }\end{array}$ \\
\hline $\begin{array}{l}\text { Peron } \\
2010 \\
{[61]}\end{array}$ & $\begin{array}{l}\text { PD } \\
\text { (UK Parkinson's } \\
\text { disease Brain } \\
\text { Bank criteria) }\end{array}$ & $\begin{array}{l}\text { Not } \\
\text { specified }\end{array}$ & $\begin{array}{l}\text { STN } \\
\text { Bilat. }\end{array}$ & $\begin{array}{l}\text { Levodopa } \\
\text { equivalent daily } \\
\text { dose } \\
\quad 1081.1 \pm 605.3 \\
\text { mg }\end{array}$ & $\begin{array}{l}1 \text { pre-DBS } \\
\text { (3 months) } \\
1 \text { post-DBS } \\
\text { (3 months) }\end{array}$ & $\begin{array}{l}13 \\
53.3 \pm 8.5 \\
5 / 8\end{array}$ & $\begin{array}{l}13 ; \\
N R ; \\
5 / 8\end{array}$ & $\begin{array}{l}\text { Pre: } 31.4 \\
\pm 12.2 ; \\
\text { Post: } 14.1 \\
\pm 7.4 \text { (DBS ON) }\end{array}$ \\
\hline $\begin{array}{l}\text { Sidtis } \\
2012 \\
{[62]}\end{array}$ & $\begin{array}{l}\text { PD } \\
(N R)\end{array}$ & NR & $\begin{array}{l}\text { STN } \\
\text { Bilat. }\end{array}$ & $\begin{array}{l}\text { Levodopa } \\
450 \text { mg }\end{array}$ & $\begin{array}{l}1 \text { post-DBS } \\
\text { (mean: } 2.2 \\
\text { years) }\end{array}$ & $\begin{array}{l}7 ; \\
57.1 ; \\
0 / 7\end{array}$ & - & $\begin{array}{l}\text { Pre: NR } \\
\text { Post: } 35.6 \\
\text { (DBS OFF) } \\
\text { Pre: } 57.3 \\
\pm 15.3\end{array}$ \\
\hline $\begin{array}{l}\text { Smith } \\
2019 \\
{[63]}\end{array}$ & $\begin{array}{l}\text { PD } \\
\text { (UK Parkinson's } \\
\text { disease Brain Bank } \\
\text { criteria) }\end{array}$ & $\begin{array}{l}\text { CAPSIT } \\
\text { and } \\
\text { NICE } \\
\text { criteria }\end{array}$ & $\begin{array}{l}\text { STN } \\
\text { Bilat. }\end{array}$ & Levodopa & $\begin{array}{l}1 \text { pre-DBS } \\
1 \text { post-DBS } \\
\text { (mean: } 5 \\
\text { months) }\end{array}$ & $\begin{array}{l}7 \\
66 \pm 7 \\
3 / 4\end{array}$ & - & $\begin{array}{l}\text { Post: } 37.6 \pm \\
20 \\
\text { (DBS ON) } \\
54.6 \\
\text { (DBS OFF only } \\
3 \text { patients) }\end{array}$ \\
\hline $\begin{array}{l}\text { Vassal } \\
2019 \\
{[64]}\end{array}$ & $\begin{array}{l}P D \\
\text { (NR) }\end{array}$ & $\begin{array}{l}\text { Not } \\
\text { specified }\end{array}$ & $\begin{array}{l}\text { STN } \\
\text { Bilat. }\end{array}$ & $\begin{array}{l}\text { Levodopa } \\
\text { equivalent } \\
\text { daily dose } \\
1497 \pm 364.5 \\
\text { mg }\end{array}$ & $\begin{array}{l}1 \text { pre-DBS } \\
2 \text { post-DBS } \\
\text { ( } 3 \text { months; } \\
6 \text { months) }\end{array}$ & $\begin{array}{l}9 ; \\
58 \pm 6.3 \\
4 / 5\end{array}$ & - & $\begin{array}{l}\text { Pre: } 33.8 \\
\pm 10.6 \\
\quad \text { Post: } 17 \pm 5.5 \\
\text { (DBS ON) }\end{array}$ \\
\hline
\end{tabular}


NR (Not reported); - (Absent); DBS-PD (patients with PD treated with DBS); MT-PD (patients with PD treated only pharmacologically); HC (Healthy controls); UPDRS (Unified Parkinson's Disease Rating Scale); STN (Subthalamic nucleus); GPi (Globus Pallidus internus); VIM (Ventral Intermediate nucleus of the thalamus); Bilat. (Bilaterally); FOG (Freezing of Gait)

Table 2a Summary of the analytical procedures and results of the biochemical and epigenetic studies.

\begin{tabular}{|c|c|c|c|c|c|c|c|c|}
\hline Study & Specimen & Sample Processing & $\begin{array}{l}\text { Analytical } \\
\text { procedure } \\
\text { of the } \\
\text { sample }\end{array}$ & Biomarker & $\begin{array}{l}\text { Level of bioma } \\
\text { VS Controls } \\
1^{\text {st }} \\
\text { follow- } \\
\text { up }\end{array}$ & $\begin{array}{l}2^{\text {nd }} \\
\text { follow- } \\
\text { up }\end{array}$ & $\begin{array}{l}3^{\text {rd }} \\
\text { follow- } \\
\text { up }\end{array}$ & $\begin{array}{l}4^{\text {th }} \\
\text { follow } \\
\text { - } \\
\text { up }\end{array}$ \\
\hline \multicolumn{9}{|l|}{ BIOCHEMICAL } \\
\hline $\begin{array}{l}\text { Constantinescu } \\
2011 \\
{[22]}\end{array}$ & CSF & Storage & ELISA & NFL & $\begin{array}{l}\text { 个 NFL } \\
\text { Levels }\end{array}$ & $\begin{array}{l}\text { 个FL } \\
\text { levels }\end{array}$ & $\begin{array}{l}\downarrow N F L \\
\text { levels }\end{array}$ & $\begin{array}{l}\downarrow N F L \\
\text { levels }\end{array}$ \\
\hline $\begin{array}{l}\text { Constantinescu } \\
2018 \\
{[23]}\end{array}$ & CSF & Storage & ELISA & $\begin{array}{l}\text { NFL, T-Tau, } \\
\text { p-Tau, GFAP, } \\
\text { A } 342\end{array}$ & $\begin{array}{l}\text { 个 NFL, } \\
\text { t-Tau. } \\
\text { GFAP } \\
\text { levels }\end{array}$ & $\begin{array}{l}\text { 个NFL, } \\
\text { t-Tau, } \\
\text { GFAP } \\
\text { levels }\end{array}$ & $\begin{array}{l}\downarrow N F L \\
\text { t-TAU, GFAP } \\
\text { levels }\end{array}$ & $\begin{array}{l}\downarrow N F L \\
\text { t-Tau, } \\
\text { GFAP } \\
\text { levels }\end{array}$ \\
\hline $\begin{array}{l}\text { Dong } \\
2019 \\
{[24]}\end{array}$ & Plasma & Centrifuge & $\begin{array}{l}\text { Tandem } \\
\text { mass tag } \\
\text { markers and } \\
\text { liquid } \\
\text { chromatogra } \\
\text { phy-mass } \\
\text { spectrometr- } \\
\text { based }\end{array}$ & $\begin{array}{l}\text { CCDC154, } \\
\text { TRIM3, DHH, } \\
\text { NRP2, CLIC1 }\end{array}$ & $\begin{array}{l}\downarrow \text { expression } \\
\text { of CCDC154, } \\
\text { TRIM3, NHH } \\
\uparrow \text { expression } \\
\text { of } \\
\text { NRP2, CLIC1 }\end{array}$ & - & - & - \\
\hline
\end{tabular}


techniques

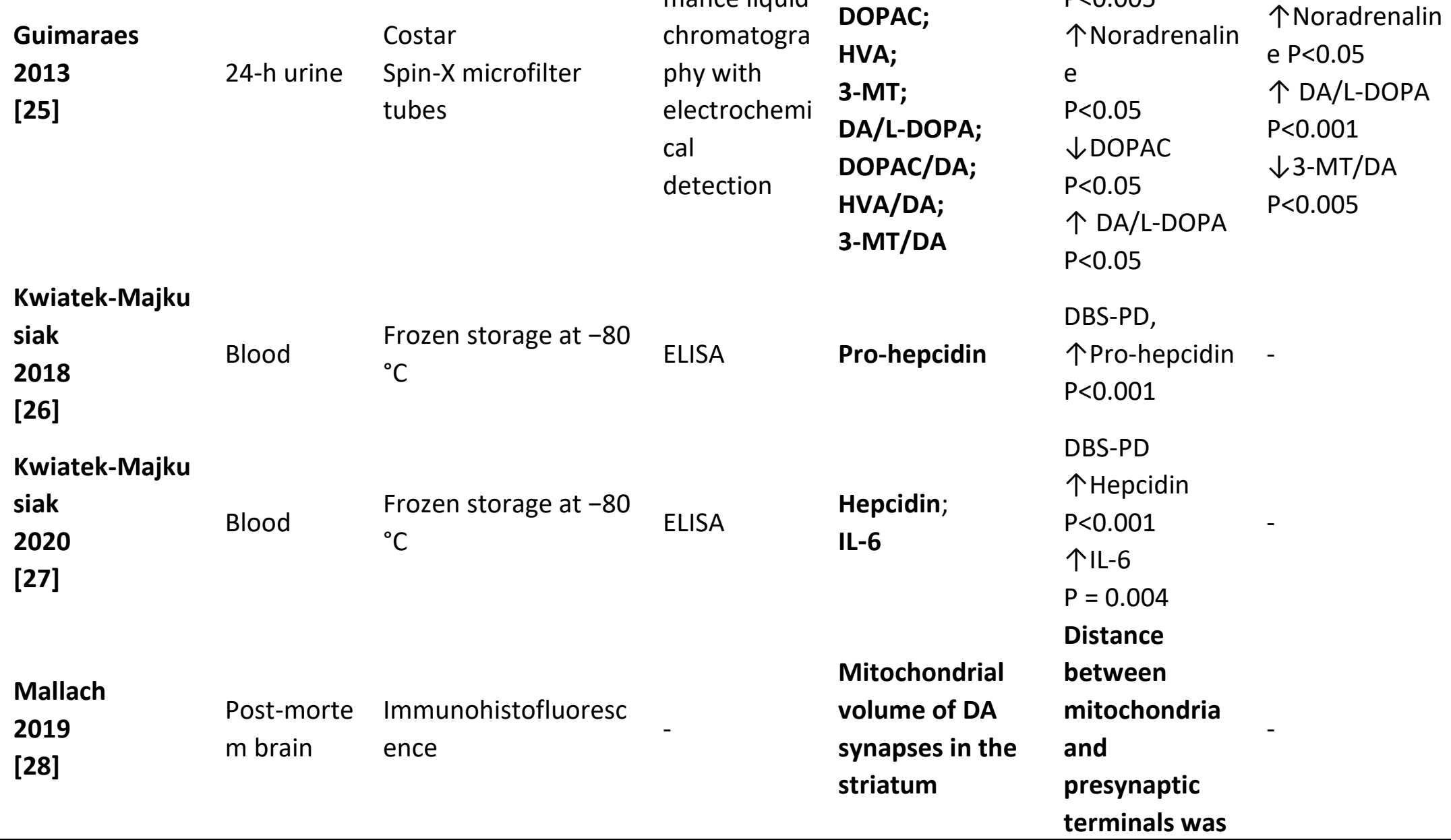




\section{Pal}

2017

[29]

Post-morte Immunohistofluoresc

$\mathrm{m}$ brain

ence

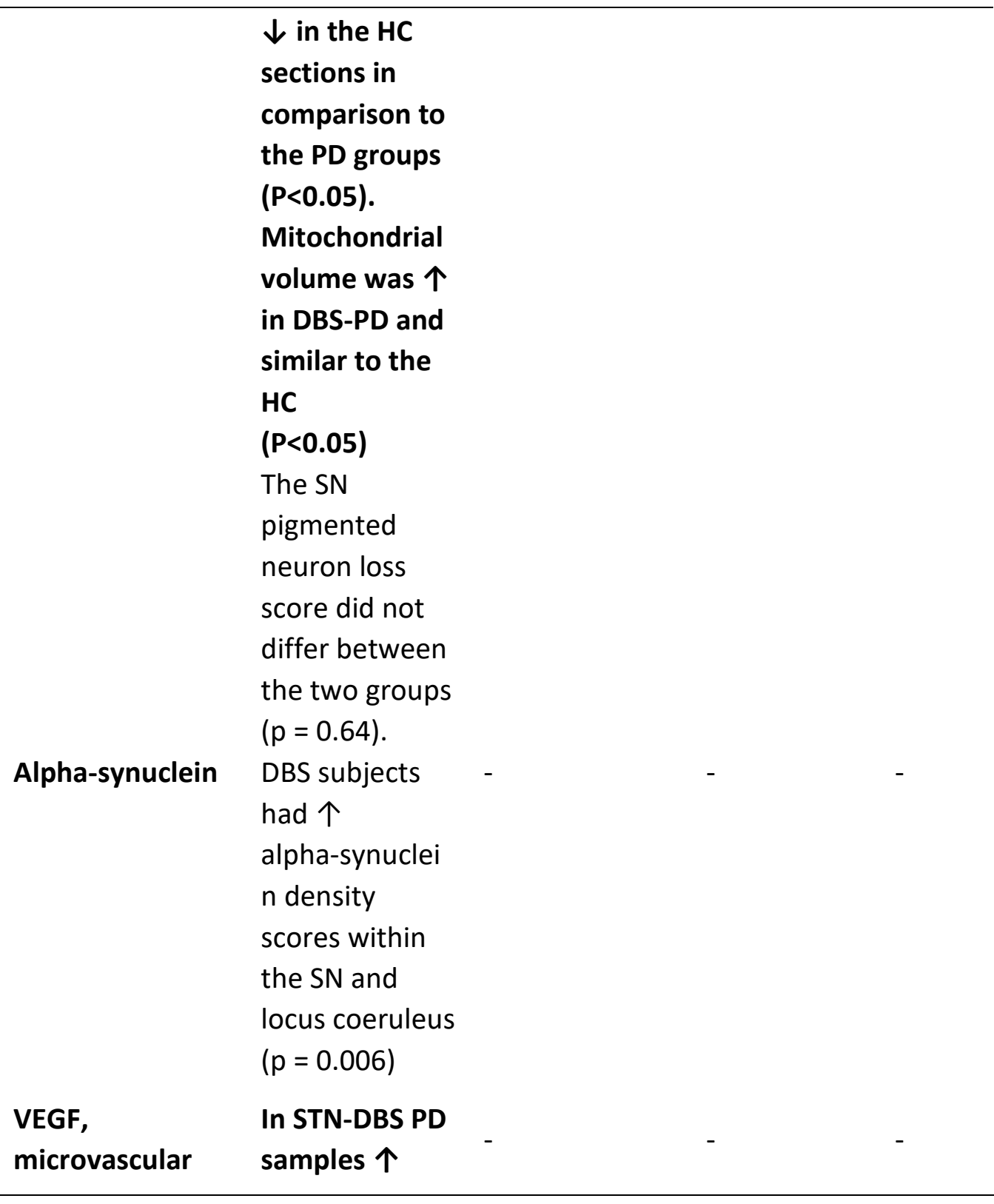

comparison to

the PD groups

in DBS-PD and

similar to the

The SN

pigmented

neuron loss

score did not

differ between

two groups

had $\uparrow$

alpha-synucle

n density

scores within

SN and

Post-morte Histopathology,

$\mathrm{m}$ brain

immunofluorescence

microvascular 


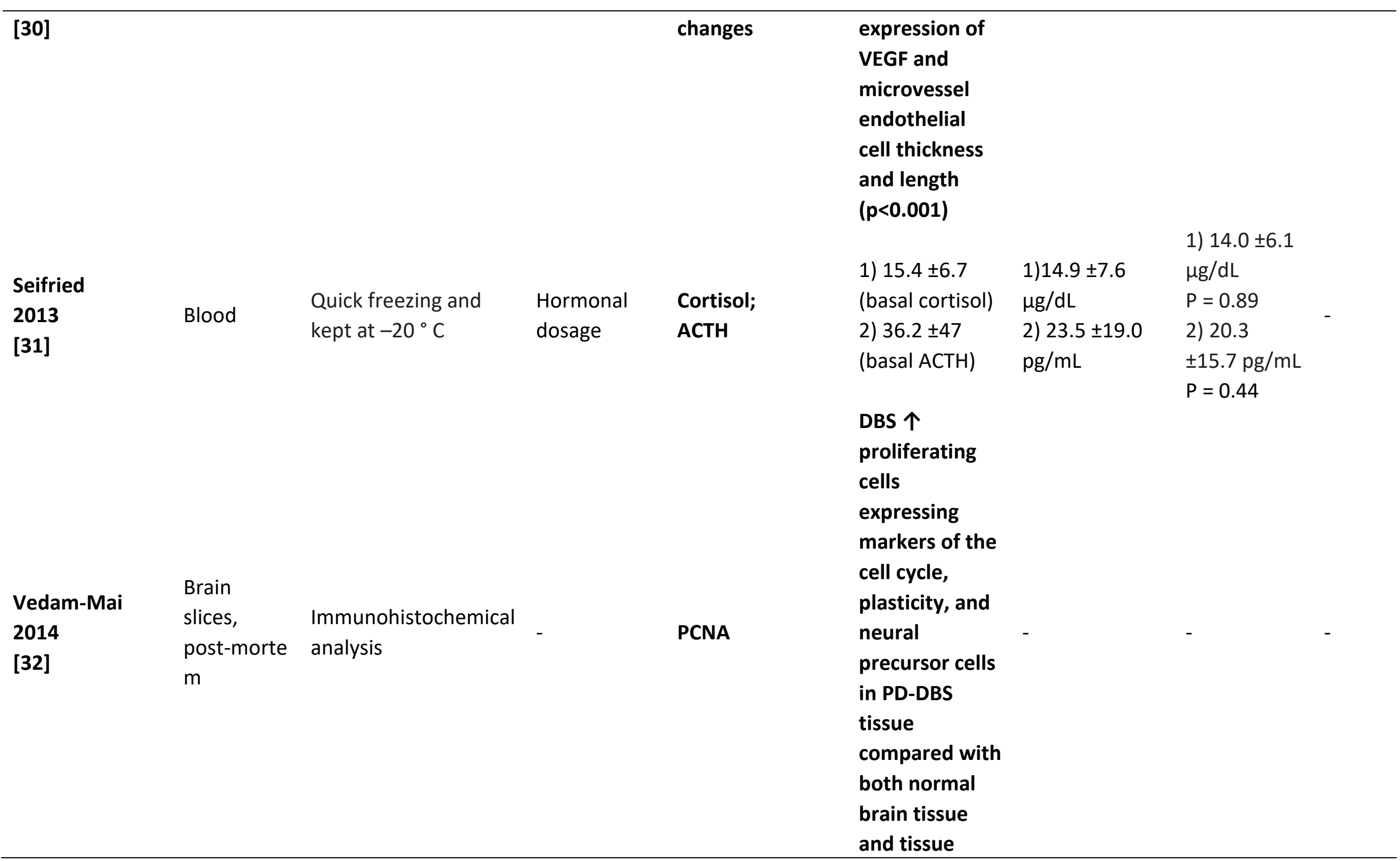




\begin{tabular}{|c|c|c|c|c|c|c|c|}
\hline & & & & $\begin{array}{l}\text { from patients } \\
\text { with PD not } \\
\text { treated with } \\
\text { DBS (P<0.05). } \\
\text { The level of } \\
\text { cell } \\
\text { proliferation in } \\
\text { the SVZ in } \\
\text { PD-DBS brains } \\
\text { was 2-6 fold } \\
\text { greater than } \\
\text { that in normal } \\
\text { and untreated } \\
\text { PD brains }\end{array}$ & & & \\
\hline $\begin{array}{l}\text { Wang } \\
2013 \\
{[33]}\end{array}$ & Centrifuge & $\begin{array}{l}\text { 2-D DIGE in } \\
\text { combination } \\
\text { with } \\
\text { MALDI-TOF } \\
\text { and TOF-TOF } \\
\text { mass } \\
\text { spectrometr } \\
\text { y; Western } \\
\text { Blotting }\end{array}$ & $\begin{array}{l}21 \text { different } \\
\text { proteins such as: } \\
\text { apoA-1, C4, IgA, } \\
\text { EC-SOD, IgK } \\
\text { protein, myosin, } \\
\text { tetranectin }\end{array}$ & $\begin{array}{l}\text { 个EC-SOD } \\
P<0.05 \\
\uparrow \text { Tetranectin } \\
P<0.05\end{array}$ & $\begin{array}{l}\uparrow E C-S O D \\
P<0.05 \\
\uparrow T e t r a n e c t i n \\
P<0.05\end{array}$ & $\begin{array}{l}\text { } E C-S O D \\
P<0.05 \\
\uparrow \text { Tetranecti } \\
n \\
P<0.05\end{array}$ & - \\
\hline \multicolumn{8}{|c|}{ EPIGENETIC } \\
\hline $\begin{array}{l}\text { Soreq } \\
2012 \\
{[34]}\end{array}$ & $\begin{array}{l}\text { Blood } \\
\text { (leucocytes } \\
\text { ) }\end{array}$ & $\begin{array}{l}\text { Affymetrix } \\
\text { exon array }\end{array}$ & $\begin{array}{l}\text { Transcript } \\
\text { isoforms }\end{array}$ & $\begin{array}{l}173 \text { Transcripts } \\
\text { of patients } \\
\text { with PD differ } \\
\text { from controls }\end{array}$ & $\begin{array}{l}\text { Pre-DBS vs } \\
\text { post-DBS } \\
465 \text { genes } \\
\text { differentially }\end{array}$ & $\begin{array}{l}\text { The OFF DBS } \\
\text { state was } \\
\text { accompanie } \\
\text { d by }\end{array}$ & - \\
\hline
\end{tabular}




\begin{tabular}{|c|c|c|c|c|c|c|c|}
\hline & & & & 16 mipNAs & $\begin{array}{l}\text { expressed after } \\
\text { DBS surgery; } \\
\text { post-DBS vs HC } \\
321 \text { transcripts } \\
\text { changed } \\
\text { between PD } \\
\text { patients' } \\
\text { post-DBS to } \\
\text { HC, including } \\
\text { PARK7 and } \\
\text { PARK1 which } \\
\text { maintained } \\
\text { PD-characterist } \\
\text { ic changes. } \\
\text { Pre-DBS vs }\end{array}$ & $\begin{array}{l}\text { differential } \\
\text { expression } \\
\text { of } 351 \\
\text { transcripts }\end{array}$ & \\
\hline $\begin{array}{l}\text { Soreq } \\
2013 a \\
{[35]}\end{array}$ & $\begin{array}{l}\text { Blood } \\
\text { (leucocytes } \\
\text { ) }\end{array}$ & $\begin{array}{l}\text { Exon arrays } \\
\text { analysis }\end{array}$ & MiRNAs & $\begin{array}{l}16 \text { miRNAs } \\
\text { modified } \\
332 \text { changed } \\
\text { isoforms }\end{array}$ & $\begin{array}{l}\text { post-DBS } \\
11 \text { miRNAs } \\
\text { modified } \\
155 \text { changed } \\
\text { isoforms }\end{array}$ & - & - \\
\hline $\begin{array}{l}\text { Soreq } \\
2013 b \\
{[36]}\end{array}$ & $\begin{array}{l}\text { Blood } \\
\text { (leucocytes } \\
\text { ) }\end{array}$ & $\begin{array}{l}\text { In-house } \\
\text { exon array } \\
\text { leukocyte } \\
\text { dataset }\end{array}$ & $\begin{array}{l}\text { Alternative } \\
\text { Splicing } \\
\text { (AS) }\end{array}$ & $\begin{array}{l}319 \text { AS } \\
\text { changed } \\
146 \text { AS } \\
\text { changed }\end{array}$ & $\begin{array}{l}\text { Pre-DBS vs } \\
\text { post-DBS } \\
254 \text { AS } \\
\text { changes }\end{array}$ & - & - \\
\hline $\begin{array}{l}\text { Soreq } \\
2014 \\
{[37]}\end{array}$ & $\begin{array}{l}\text { Blood } \\
\text { (leucocytes } \\
\text { ) }\end{array}$ & RNA-Seq & $\begin{array}{l}\text { long non-coding } \\
\text { RNAs } \\
\text { (IncRNA) }\end{array}$ & $\begin{array}{l}\text { PD vs HC } \\
\downarrow 13 \text { IncRNA } \\
\text { expression }\end{array}$ & $\begin{array}{l}\text { DBS modified } \\
663 \text { IncRNA } \\
(18 \text { IncRNA } \\
P<0.05)\end{array}$ & - & - \\
\hline
\end{tabular}


$\uparrow$ (increased); $\downarrow$ (decreased); PD (Parkinsonian patients); HC (Healthy controls); CSF (Cerebrospinal fluid); NFL (neurofilament triplet protein); t-Tau (total-TAU); p-Tau (phosphorylated-tau); GFAP (glial fibrillary acidic protein); A -42 (brain amyloidosis); CCDC154 (coiled-coil domain-containing protein 154); TRIM3 (tripartite motif-containing protein 3); DHH (desert hedgehog protein); NRP2 (neuropilin); CLIC1 (chloride intracellular channel protein 1); IL-6 (interleukine-6); PCNA (Proliferating Cell Nuclear Antigen antibody); ApoA-1 (Apolipoprotein-A1); IgA (Immunoglobulin A); IGK (Immunoglobulin Kappa); EC-SOD (extracellular superoxide dismutase); L-DOPA (levodopa); DA (dopamine); DOPAC (3,4-dihydroxyphenylacetic acid); HVA (chemical homovanilic acid); 3-MT (3-methoxytyramine); DA/L-DOPA (ratio dopamine and levodopa); DOPAC/DA (ratio 3,4-dihydropgenylacetic acid and dopamine); MT/DA (ratio 3-methoxytyramine and dopamine); ACTH (adeno corticotropic hormone); VEGF (vascular endothelial growth factor); miRNAs (microRNAs)

Table $\mathbf{2 b}$ Summary of the analytical procedures and results of the neurophysiological studies.

\begin{tabular}{|c|c|c|c|c|c|c|c|}
\hline Study & $\begin{array}{l}\text { Type of } \\
\text { neurophysiologica } \\
\text { I procedure }\end{array}$ & $\begin{array}{l}\text { DBS condition } \\
\text { during } \\
\text { procedure } \\
\text { (ON/OFF) }\end{array}$ & Cerebral areas/pathways & $\begin{array}{l}\text { Type } \\
\text { analyzed } \\
\text { activity }\end{array}$ & $\begin{array}{l}\text { Brain activity after } \\
\text { VS Controls } \\
\mathbf{1}^{\text {st }} \\
\text { follow- } \\
\text { up }\end{array}$ & $\begin{array}{l}2^{\text {st }} \\
\text { follow- } \\
\text { up }\end{array}$ & $\begin{array}{l}3^{\text {rd }} \\
\text { follow- } \\
\text { up }\end{array}$ \\
\hline \multicolumn{8}{|c|}{ ELECTROPHYSIOLOGICAL } \\
\hline $\begin{array}{l}\text { Airaksinen } \\
2012 \\
{[38]}\end{array}$ & $\begin{array}{l}\text { Spontaneous MEG } \\
\text { activity in the } \\
\text { somatomotor } \\
\text { (mu) and occipital } \\
\text { regions (alpha) }\end{array}$ & ON/OFF & $\begin{array}{l}\text { Somatomotor and } \\
\text { occipital regions }\end{array}$ & $\begin{array}{l}\text { Spontaneous } \\
\text { activity of } \\
\text { somatomotor } \\
\text { regions. } \\
\text { Occipital region: } \\
\text { frequency band } \\
\text { around the peak } \\
\text { alpha frequency } \\
\pm 2 \mathrm{~Hz}\end{array}$ & $\begin{array}{l}\text { Alpha peak range } \\
\text { varied between } \\
5.68 \mathrm{~Hz} \text { and } 10.87 \\
\mathrm{~Hz} \text {. } \\
\text { Source strength } \\
\text { decreased from } 7.6 \\
\text { to } 7.1 \mathrm{nAm} \\
\mathrm{p}=0.05\end{array}$ & - & - \\
\hline
\end{tabular}




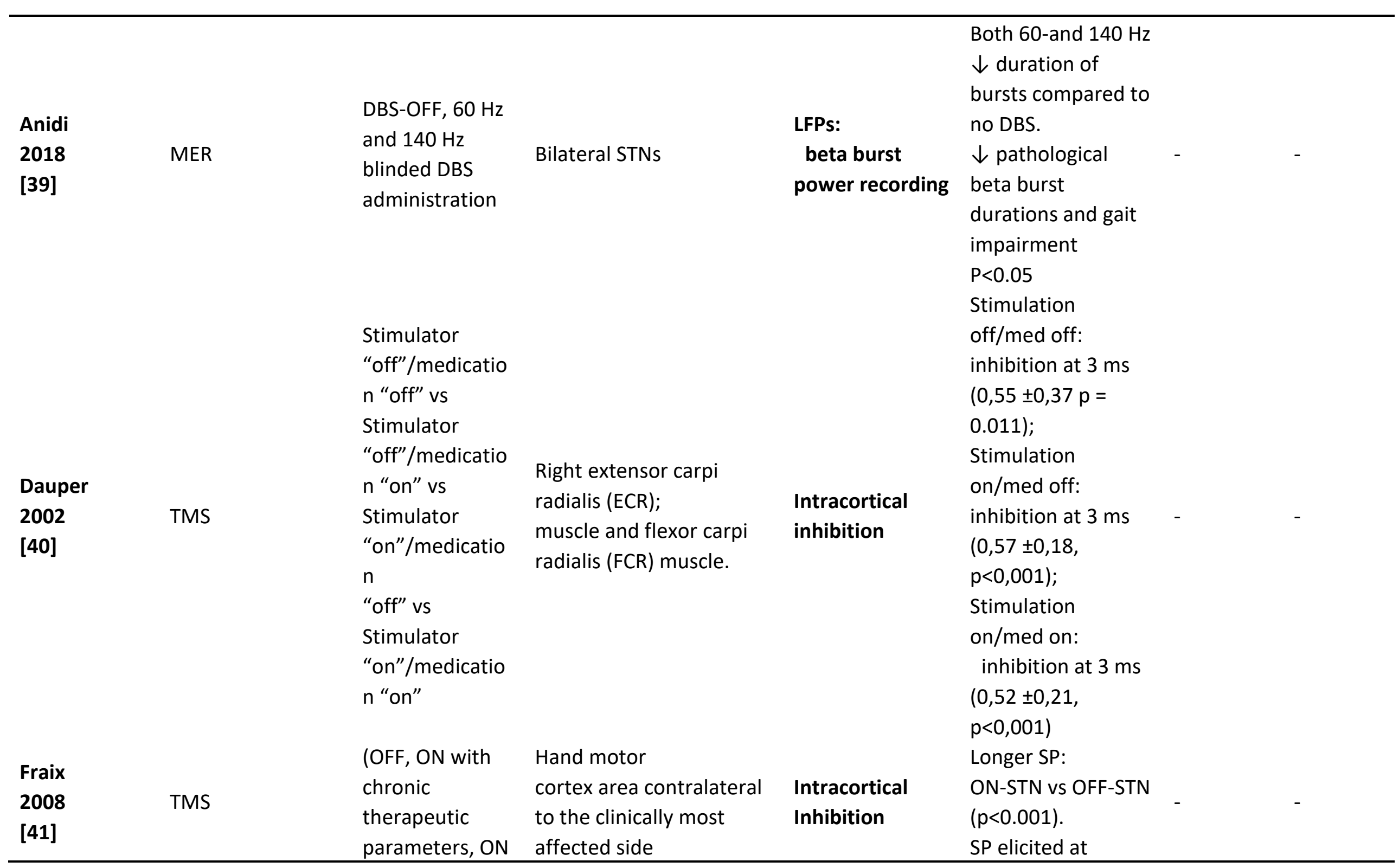




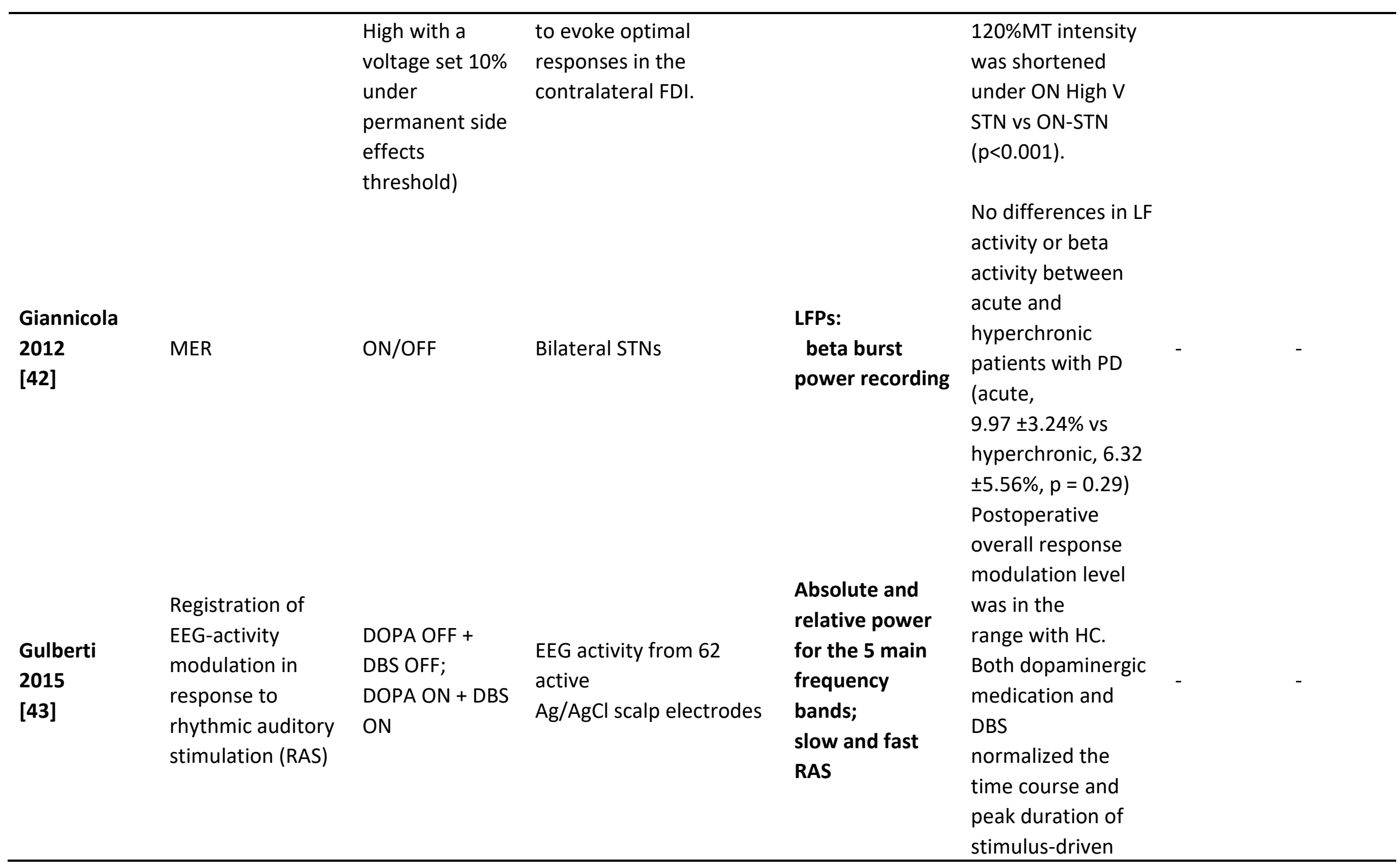


beta

power fluctuations

in the fast RAS

condition.

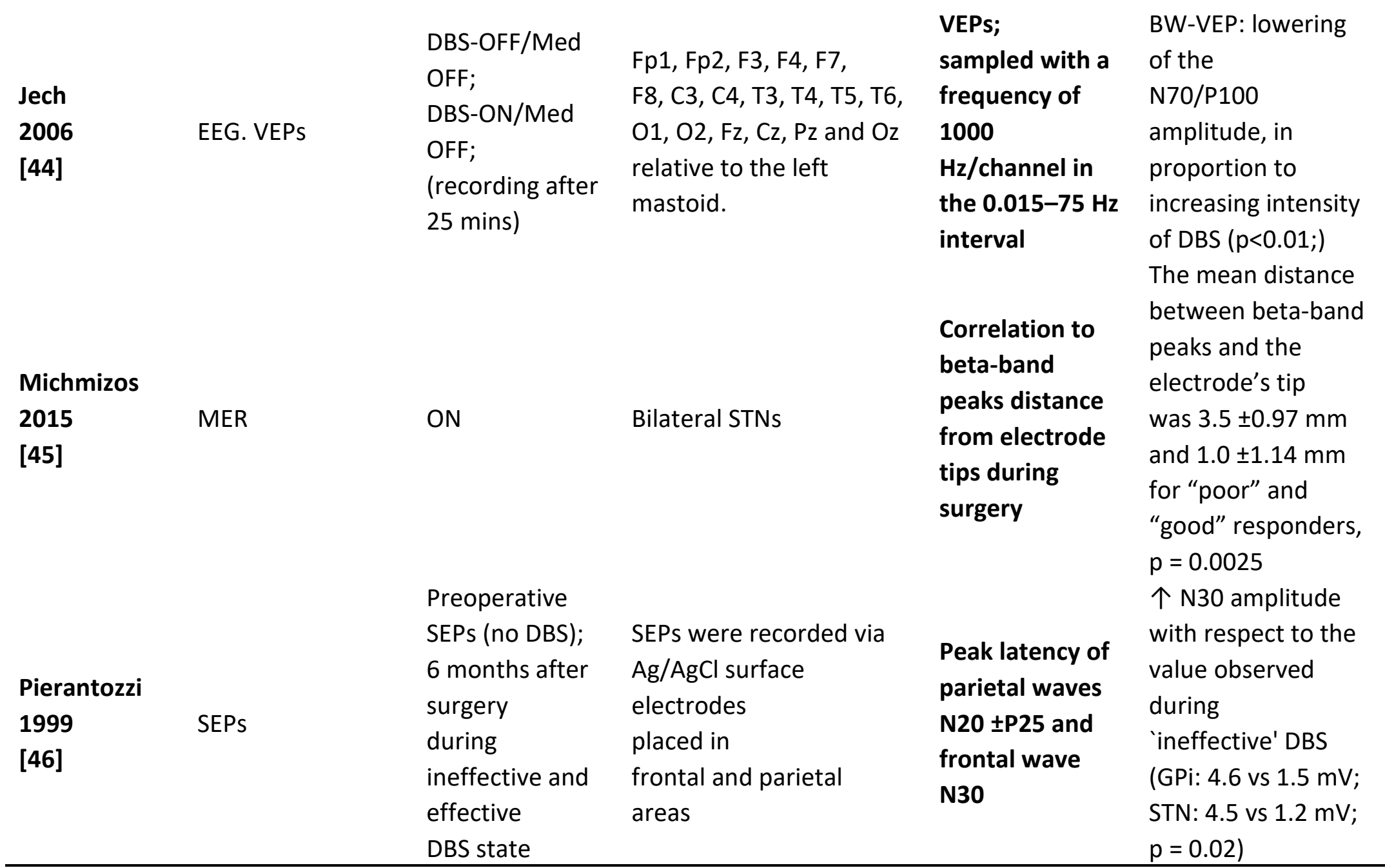




\begin{tabular}{|c|c|c|c|c|c|c|c|}
\hline $\begin{array}{l}\text { Ray } \\
2008 \\
{[47]}\end{array}$ & MER & ON/OFF & Bilateral STNs & $\begin{array}{l}\text { LFPs: } \\
\text { beta burst } \\
\text { power recording }\end{array}$ & $\begin{array}{l}\downarrow \text { beta power } \\
\text { correlated with } \\
\text { changes } \\
\text { bradykinesia/rigidit } \\
\text { y UPDRS } \\
\text { p = } 0.05 . \\
\text { Bradykinesia/rigidit } \\
\text { y } \\
\text { improvements } \\
\text { predict } \\
\text { improvements in } \\
\text { bradykinesia/rigidit } \\
\text { y after DBS, p = } \\
0.05 \\
\text { No changes in DBS }\end{array}$ & - & - \\
\hline $\begin{array}{l}\text { Rosa } \\
2011 \\
{[48]}\end{array}$ & MER & ON/OFF & STN & $\begin{array}{l}\text { LFPs: } \\
\text { beta burst } \\
\text { power recording }\end{array}$ & $\begin{array}{l}\text { off condition beta } \\
\text { activity between } \\
\text { the hyperacute and } \\
\text { chronic phases }\end{array}$ & - & - \\
\hline $\begin{array}{l}\text { Sinclair } \\
2018 \\
{[49]}\end{array}$ & MER & $\begin{array}{l}\text { ON/OFF. } \\
\text { chronic } \\
\text { stimulation was } \\
\text { ceased } 45 \text { min } \\
\text { prior to baseline } \\
\text { "off-therapy" } \\
\text { assessments. }\end{array}$ & STN & ERNA & $\begin{array}{l}\text { } \text { Dorsal STN ERNA } \\
\text { than all other } \\
\text { regions } \\
(<0.001)\end{array}$ & - & - \\
\hline $\begin{array}{l}\text { Trager } \\
2016\end{array}$ & $\begin{array}{l}\text { LFPs } \\
\text { were recorded }\end{array}$ & ON/OFF & STN & $\begin{array}{l}\text { resting state } \\
\text { LPFs: beta burst }\end{array}$ & $\begin{array}{l}\downarrow \text { beta band } \\
\text { spectra in both }\end{array}$ & $\begin{array}{l}\downarrow \text { beta } \\
\text { band }\end{array}$ & $\begin{array}{l}\downarrow \text { in beta } \\
\text { power }\end{array}$ \\
\hline
\end{tabular}




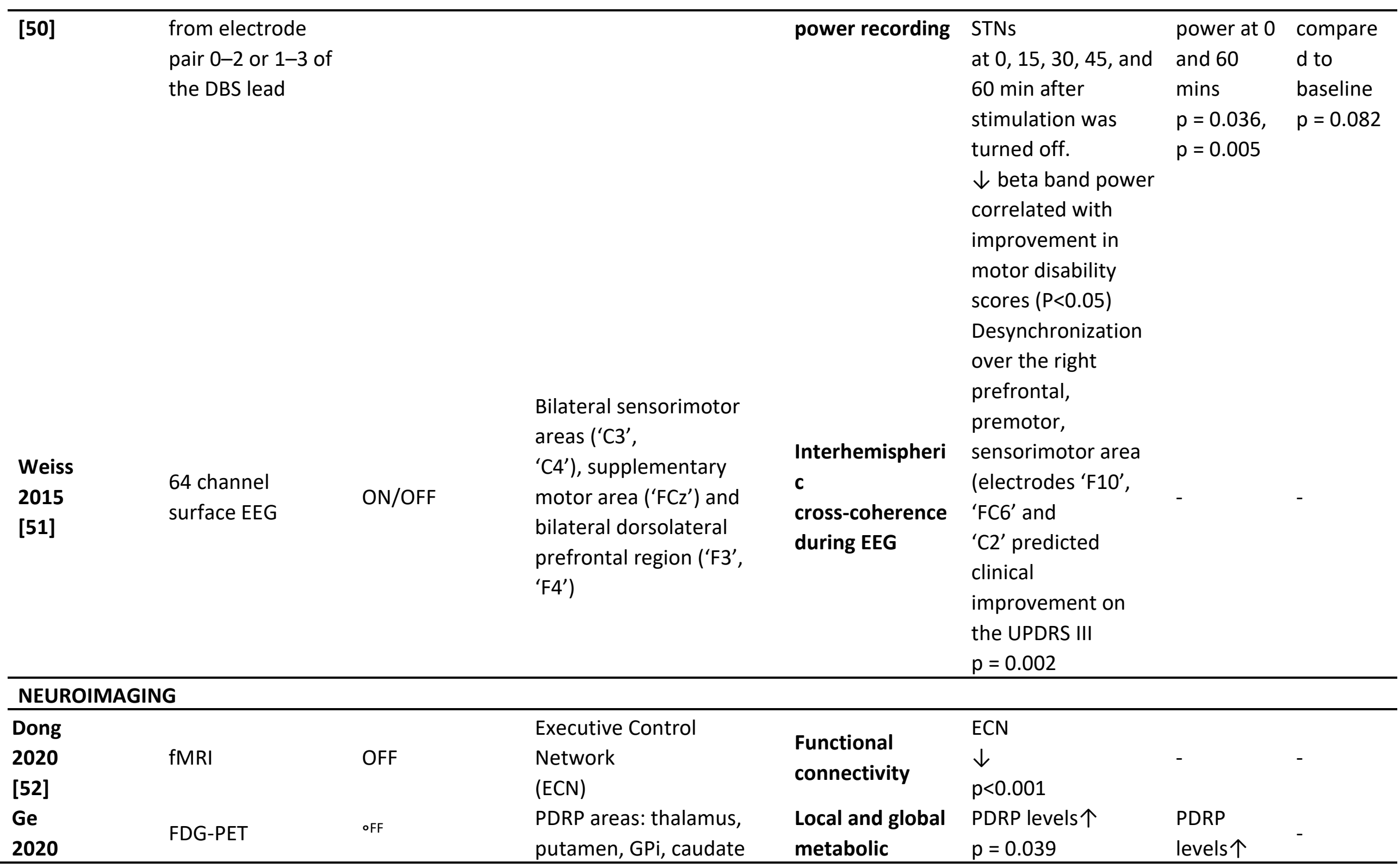




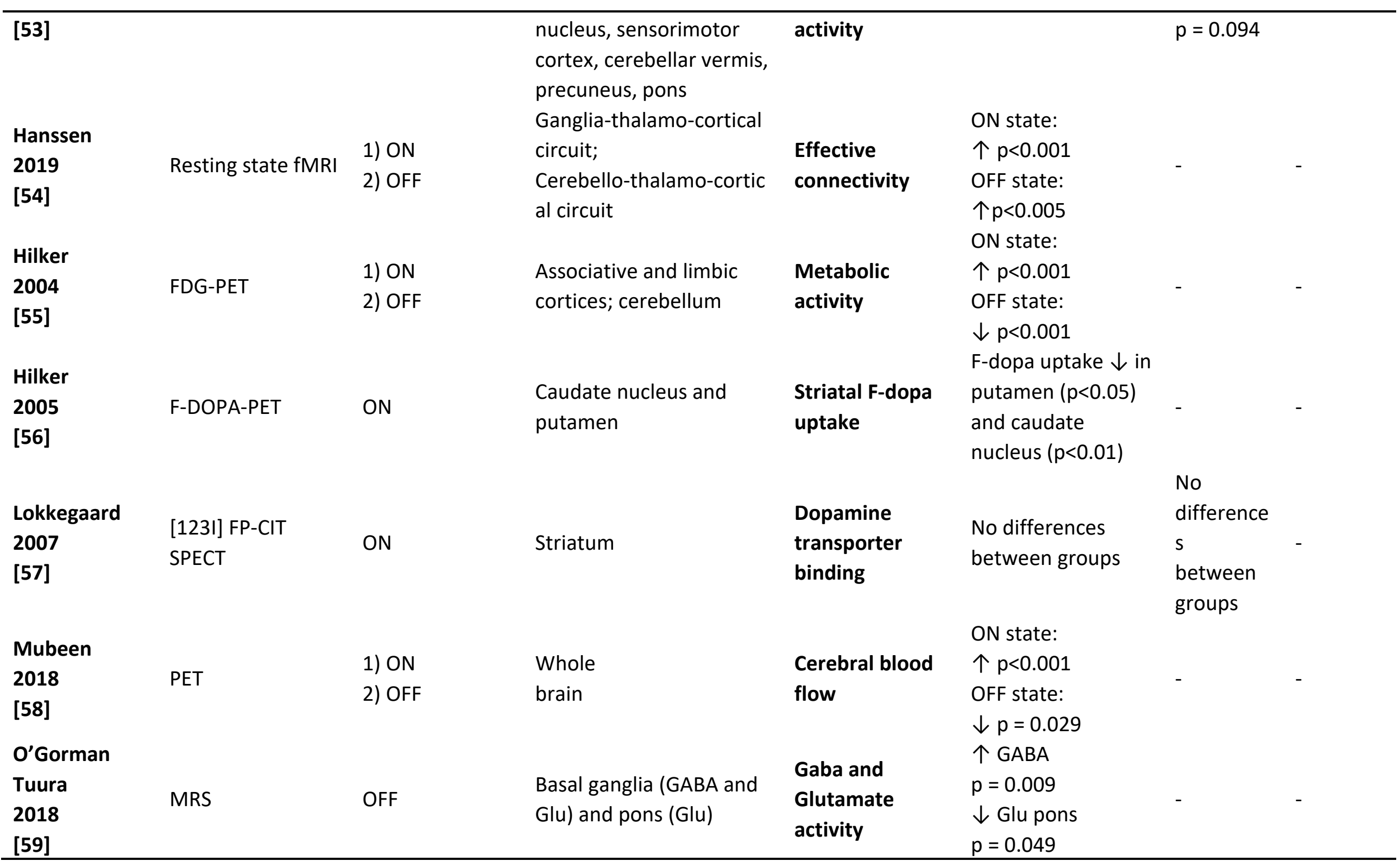




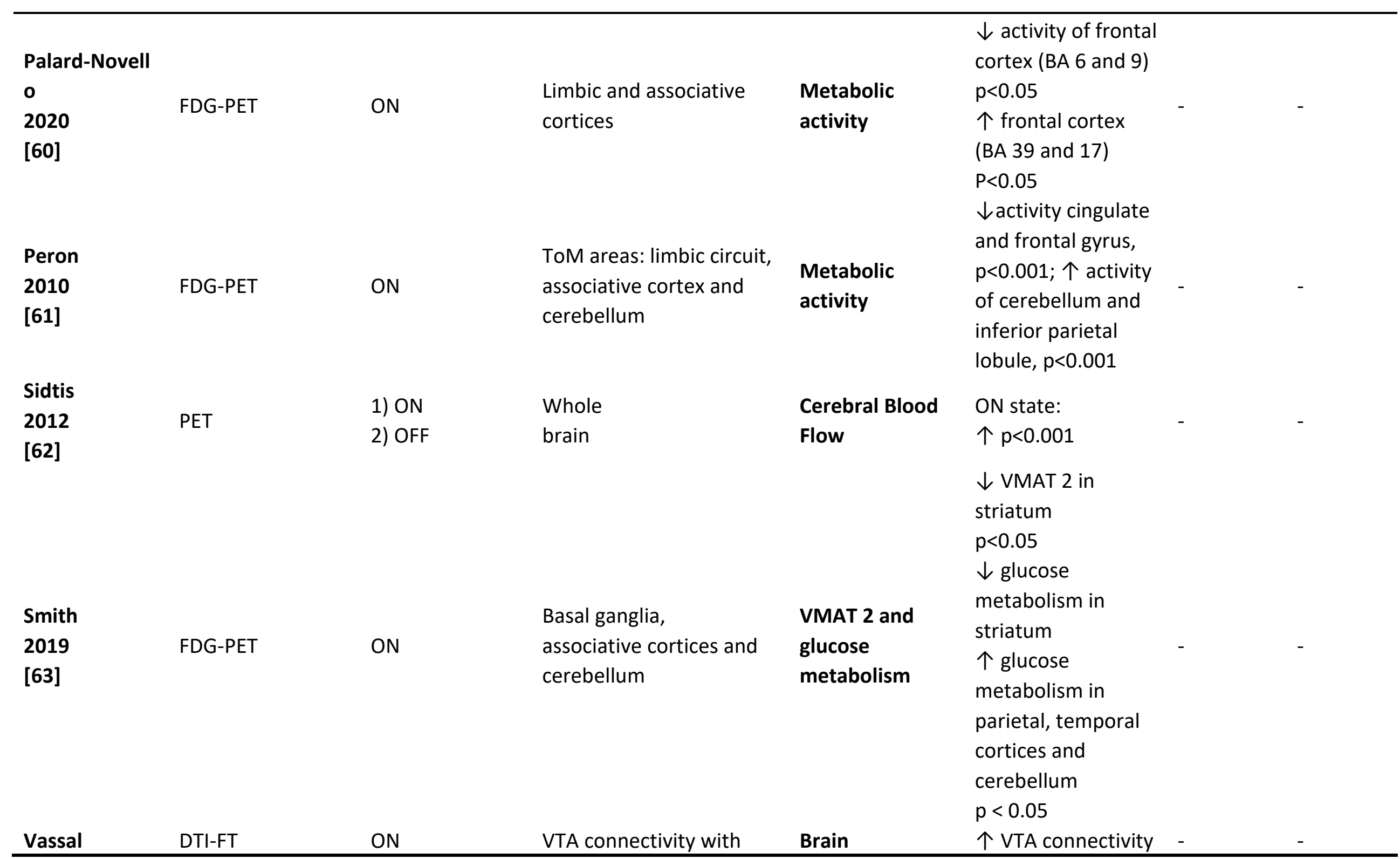




\begin{tabular}{|c|c|c|c|}
\hline $\begin{array}{l}2019 \\
{[64]}\end{array}$ & $\begin{array}{l}\text { cortical areas and } \\
\text { cerebellum }\end{array}$ & Connectivity & $\begin{array}{l}\text { with: brainstem, } \\
\text { cerebellum, } \\
\text { premotor and } \\
\text { motor cortex }\end{array}$ \\
\hline
\end{tabular}

$\uparrow$ (increased); $\downarrow$ (decreased); MER (Multi-pass microelectrode recording); TMS (Transcranial Magnetic Resonance); LFPs (Local Field Potentials); ERNA (Evoked Resonant Neural Activity); EEG (Electroencephalography); MEG (Magnetoencephalography); VEPs (Visual Evoked Potentials); SEPs (Somatosensory Evoked Potentials); STN (Subthalamic nucleus); RAS (Rhythmic auditory stimulation); FDG-PET (Fluorodeoxyglucose-Positron Emission Tomography); fMRI (functional Magnetic Resonance Imaging); [123I] FP-CIT SPECT (Single-photon emission computed tomography with [123I] FP-CIT

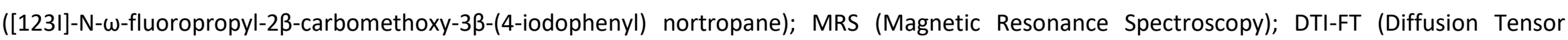
Imaging-fiber tracking); PDRP (Parkinson's Disease Related Pattern); ToM areas (Theory of Mind areas); VMAT 2 (vesicular monoamine transporter 2); VTA (Ventral Tegmental Area) 


\section{Quality Assessment}

Due to the nature of the included studies, no standardized checklists were applicable for the appraisal of the methodological quality. However, a set of predefined key qualitative elements that represented the methodological quality of the studies was established. Specifically, when assessing the selection bias, we considered how patients with PD and controls were enrolled, including the diagnostic criteria (Table 1). Diagnostic criteria and other clinical data, such as surgical criteria or Unified Parkinson's Disease Rating Scale (UPDRS) score, were also collected to characterize the study population and observe if any clinical information was relevant in explaining DBS outcomes. On the other hand, when assessing the appropriateness of the measurement of exposure, we considered the criteria applied for referring patients to DBS and the criteria for the assessment of the appropriateness of biomarker measurement (methods of sample collection, handling, storage, and analysis) (Table 2a, Table 2b). Moreover, since the disease-modifying outcomes are strictly linked with time, the time from surgery to biomarker assessment was also considered ("follow-up", Table 1) to evaluate if the length and the number of follow-ups were consistent with the biological plausibility.

\section{Results}

The bibliographic search yielded 2,960 records. A total of 75 studies were initially selected. Six duplicates were removed, and the remaining 69 studies were assessed for inclusion in the study. Later, 26 studies were excluded because they did not meet the predefined inclusion criteria. Finally, 43 studies were included for the data extraction. The process of study selection is presented in Figure 1. 


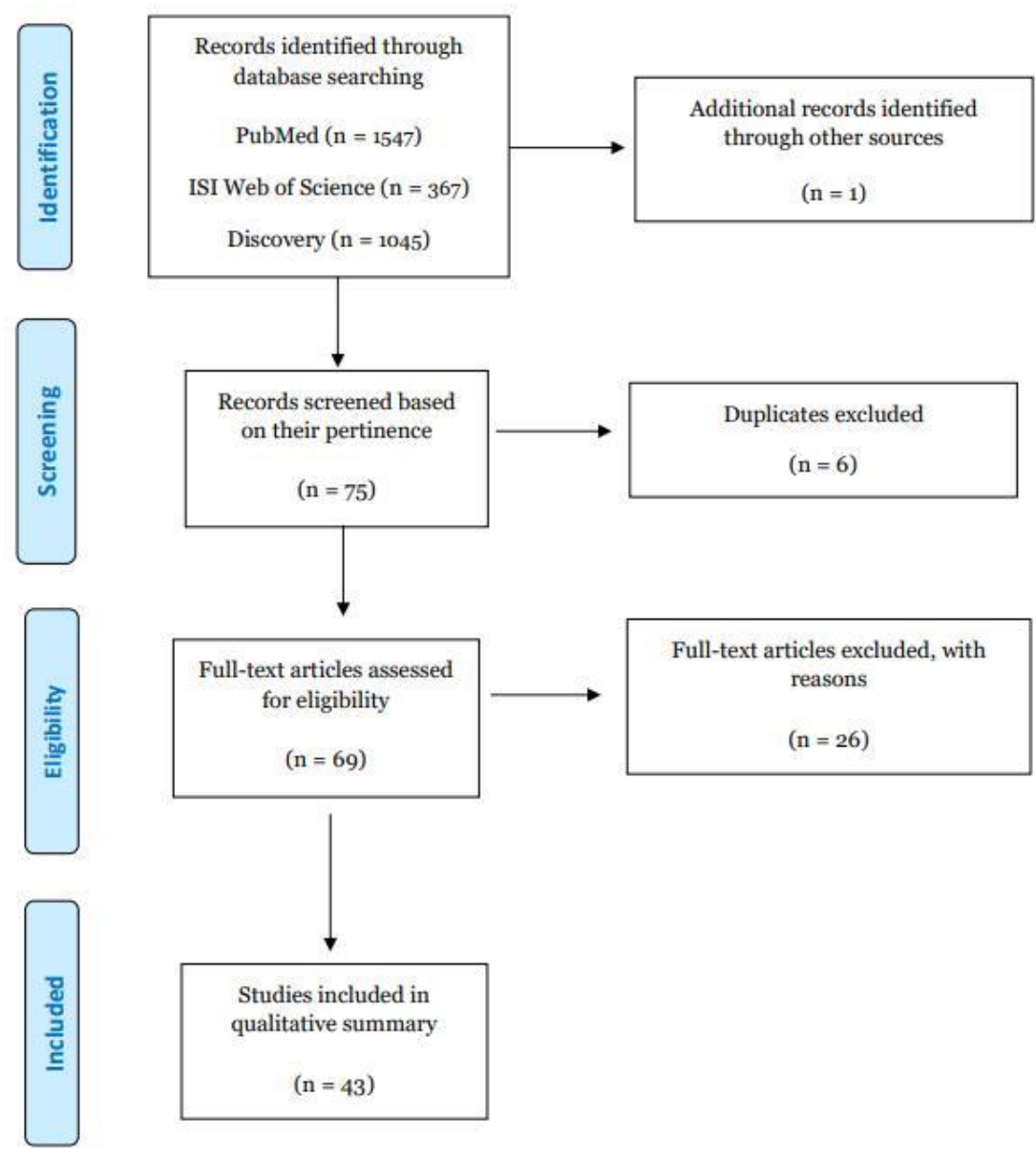

Figure 1 PRISMA flowchart describing the inclusion and exclusion of the articles

The included articles were divided into three main categories depending on the nature of DBS outcomes examined in the study: biochemical (12 studies), epigenetic (4 studies), and neurophysiological (27 studies: 14 electrophysiological and 13 neuroimaging studies).

Overall, the included studies were of moderate to low quality. As described more thoroughly in the next section, the diagnostic and surgical criteria adopted within the included studies were widely heterogeneous, and in several cases, were either not reported or not specified. Most of the studies had a small sample size and thus had limited statistical power. Moreover, examined biomarkers and analytical methods were highly heterogeneous.

\subsection{Characteristics of the Included Studies}

\subsubsection{Sample Size}

A total of 20 out of 43 studies (46.5\%) examined the effects of DBS on patients with PD without a control group. Seven of these studies had a sample size of $\leq 10$ patients, with a mean sample size of 8.3 patients. The remaining thirteen studies had enrolled a mean of 17.4 patients, with only five studies $[25,51,54,56,60]$ enrolling $\geq 20$ participants (the highest number of participants was 32 ).

The twenty-three studies that also had a control group enrolled a mean of 17 (the highest number of cases was 60) patients with PD (cases) and 18.5 controls (the highest number of 
controls was 156). Only five studies $[29,39,42,45,57]$ also included patients with PD in the control group, whereas the remaining eighteen studies enrolled healthy controls (HC).

\subsubsection{Mean Age and Gender Distribution of the Participants}

The mean age of the participants enrolled in the studies ranged from 51 to 80 years in the cases and 57 to 77 years in the controls, with 20 studies (46.5\%) recruiting PD patients with a mean age of $\leq 60$ years and no study enrolling participants aged $\leq 50$ years.

Twenty-eight studies (65.1\%) recruited a higher number of male cases (mean: 10.5 males, 5.2 females). Seven studies [28, 29, 45-47, 54, 57] did not specify the male/female ratio. Twelve studies out of 23 (52.1\%) recruited more males in the control group (mean: 7.3 males, 3.6 females), while four studies $[28,29,39,45]$ did not report the gender proportion.

\subsubsection{Diagnostic Criteria for PD}

Eleven studies [22, 26-28, 31, 53, 55, 56, 60, 61, 63] used the "UK Parkinson's disease Brain Bank criteria" [65] for the diagnosis of idiopathic PD, four studies [41, 43, 45, 46] adopted the "Hoehn and Yahr scale" [66], and two studies [24, 54] used the "Movement Disorder Society criteria" [67]. However, most of the studies (55.8\%) did not specify the adopted criteria and only reported that the cases had a neurological history of idiopathic PD.

\subsubsection{Surgical Criteria}

Twenty-eight studies (65.1\%) did not specify the surgical criteria that were adopted to select the patients with PD that could undergo DBS surgery. Four studies [45, 56, 57, 63] adopted the "CAPSIT criteria", while three studies $[43,46,51]$ used the "Hoehn and Yahr scale". However, most studies specified that PD patients that had undergone DBS surgery were refractory to medical treatment.

\subsubsection{Pharmacological Treatments}

L-DOPA was the main pharmacological treatment used to manage the motor symptoms in patients with PD (Table 1). The mean dose ranged from $300 \mathrm{mg}$ to $2000 \mathrm{mg}$ per day. Treatment was often suspended before surgery and the washout period lasted from 8 to $72 \mathrm{~h}$. Only in three studies [39, 42, 57] did the controls also receive an antiparkinsonian treatment. In the study by Michmizos et al. [45], although the cases and controls were diagnosed with PD, the pharmacological treatment was not described.

\subsubsection{UPDRS Score before Surgery}

Twenty-eight studies (65.1\%) reported the UPDRS score at baseline before the surgery. The mean UPDRS score in the OFF phase (without pharmacological treatment) ranged from 17 to 74. Eighteen studies (64.2\%) reported a score of $\geq 42$, indicating severe PD. Only in three studies by Soreq et al. [34-36] was the baseline UPDRS score <30, thus indicating moderate PD. Two studies by Anidi and Michmizos [39, 45] were the only studies that assessed the UPDRS scale in the control group, with scores of 35.7 and 59.4, respectively. 


\subsubsection{UPDRS Score after DBS}

Twenty-five studies (58.1\%) reported the UPDRS score after surgery in the cases. Only eleven studies assessed the UPDRS scale in the OFF phase (without stimulation), reporting a mean score ranging from 27.4 to 54.6. Only one [53] study reported a UPDRS score of $<30$, with a progressive increase nine months after surgery (mean score $=49.3 \pm 18.2$ ). Sixteen studies reported a mean UPDRS score in the ON phase (with DBS stimulation) ranging from 9.2 to 37.6 , with nine of them (56.2\%) reporting a score of $\leq 20$. Four studies $[47,50,54,57]$ did not specify the UPDRS score but only reported an increase or a decrease in the score.

\subsubsection{Location of Implanted DBS}

In all the studies except one [60], the electrodes were inserted bilaterally in the STN. Only in the study by Palard-Novello et al. [60] were the electrodes inserted bilaterally in the GPi. In one study [46], four patients were implanted with a bilateral GPi-DBS and two with a bilateral STN-DBS. In another study [32], seven patients had a history of bilateral stimulation in the STN, one patient had unilateral STN stimulation, while two patients had bilateral GPi stimulation, and one patient had unilateral GPi stimulation. In one study [24], the location of the electrodes was not specified. Only in three studies $[39,42,45]$ was the control group also treated with DBS.

\subsubsection{Follow-Ups}

In 20 out of 43 studies (46.5\%), the participants were observed before DBS and after several months after surgery. The mean duration of follow-up after DBS surgery ranged from 1 month to 11 years. However, only Constantinescu et al. [23] reported a follow-up of 11 years, while 26 out of 43 studies (60.4\%) followed up the participants for only $\leq 1$ year.

Eleven studies (26.8\%) enrolled patients with PD that had already received an implant and had been treated with DBS for at least 1 year.

Four post-mortem studies $[28,29,30,32]$ examined the brain tissue of patients with PD who had been treated with DBS for a mean of five years until death.

Moreover, 14 out of 43 studies (32.5\%) followed up with participants at least twice after DBS, whereas the remaining 29 studies (67.4\%) observed patients once after DBS, with most being neurophysiological studies (75.9\%). On the other hand, five biochemical [22, 23, 25, 31, 33] and all the epigenetic studies followed-up participants at least twice after the surgical procedure.

\subsection{Biochemical Studies}

We included twelve studies that assessed the biochemical changes in PD patients that had undergone DBS treatment to explore if the surgery modified the progression of PD by changing the biochemical patterns.

As described in the introduction, one of the main biochemical hallmarks of PD is the aggregation of Lewy bodies composed of $\alpha$-synuclein. However, only one study [29] investigated the changes in $\alpha$-synuclein after exposure to DBS. That study had focused on the post-mortem brain tissue of patients with PD by assessing $\alpha$-synuclein and SN neurons. The authors did not observe significant results suggesting a potential disease-modifying effect of surgery. Rather, they 
showed an increase in the density of $\alpha$-synuclein after DBS, suggesting a progression of the disease.

In contrast, three other post-mortem studies [28, 30,32] reported a significant effect of DBS on the brain of treated participants compared to those who did not receive DBS and HC. The studies focused on different biomarkers, such as the mean distance and mitochondrial volume of dopaminergic synapses in the striatum [28], Vascular Endothelial Growth Factor (VEGF) [64], and Proliferating Cell Nuclear Antigen antibody (PCNA) in the sub-ventricular zone (SVZ) [31]. Overall, the results of these studies indicated a remarkable neuroprotective action of DBS through neurotrophic mechanisms.

Moreover, since tau proteins and brain amyloidosis $(A \beta-42)$ are important markers of neurodegenerative processes, one study [23] investigated the potential association between the levels of cerebrospinal fluid (CSF) and the exposure to DBS. Those authors did not find any significant results throughout the follow-up, suggesting that DBS might have a minimal effect on these neurodegenerative mechanisms.

On the other hand, the study by Wang et al. [33] reported an interesting result on the potential disease-modifying effect of DBS. The authors found a link between tetranectin and STN stimulation. Since tetranectin is involved in the degradation of proteins in the brain, the authors suggested that elevated tetranectin levels following STN-DBS could be linked to a reduction in the aggregation of abnormal proteins and neurodegenerative processes [33].

Neurodegeneration in PD also includes chronic inflammation processes and oxidative stress, which are related to a dysregulation in the homeostasis of iron metabolism [68]. Based on this, two studies [26, 27] assessed the pre-and post-DBS levels of two proteins (pro-hepcidin and hepcidin) involved in iron metabolism and a protein linked to anti-inflammatory processes (interleukin-6), reporting an unclear link between these proteins and DBS.

The remaining four studies $[22,24,25,31]$ investigated the efficacy of DBS by assessing several biomarkers, including HPA (Hypothalamic-pituitary-adrenal) axis markers [31] and urinary levels of catecholamines [25], but they did not find any significant results supporting a disease-modifying effect.

\subsection{Epigenetic Studies}

We included four epigenetic studies by Soreq and colleagues [34-37]. In all the included trials, blood samples were collected to analyze leukocytes, adopting the same study design. However, none of these studies examined potential disease-modifying effects. The authors were initially interested in investigating any relations between molecular changes and reversible motor improvements induced by STN-DBS. The authors focused on different epigenetic biomarkers potentially involved in PD, such as transcript isoforms [34], miRNAs [35], alternative splicing (AS) events [36], and long non-coding RNA (IncRNA) [37]. In all these studies, Soreq and colleagues reported significant but transient modifications of the biomarkers in participants treated with DBS in the ON phase, suggesting that surgery may only have a reversible effect on PD symptoms. However, the potential utility of these markers in assessing the biological effects of DBS in patients with PD should be further explored, as they have been found in peripheral biofluids (saliva, blood, plasma, serum, and urine) and are thus easy to obtain with no substantial health risks. 


\subsection{Neurophysiological Studies}

\subsubsection{Electrophysiological Studies}

Parkinsonian aberrations such as rigidity have also been correlated with impairments in beta oscillations [40]. Therefore, it is important to explore changes in the beta activity, as markers of potentially relevant effects of DBS on the pathophysiology of PD. We included six electrophysiological studies [39, 42, 45, 47, 48, 50] that investigated Local Field Potentials (LFPs) focusing on beta burst activity. None of these studies found significant results suggesting a potential disease-modifying effect of DBS. Giannicola et al. [42] investigated potential disease-modifying patterns but did not observe any differences in the beta activity between acute and hyper-chronic patients with PD treated with DBS. Other studies reported only a temporary beneficial effect on the beta activity, pointing out that a decrease in the beta burst activity was associated with improved motor symptoms in patients with PD [39, 43, 45, 47, 50, 51].

The remaining studies [38, 40,41, 44, 46, 49] examined different cortical regions and waves, reporting only transient positive effects on motor symptoms after stimulation.

\subsubsection{Neuroimaging Studies}

The use of neuroimaging techniques such as PET or MRI is an accessible method to measure the efficacy of DBS and identify the possible physiological changes in the brain of treated patients.

Most of the neuroimaging studies included in this review had explored different cerebral pathways to assess a potential neurophysiological effect of DBS. Four studies [53, 56, 57, 63] investigated the potential of DBS to affect the progression of the disease through possible neuroprotective effects. None of these studies reported any significant results. Lokkegaard et al. [57] compared patients that had undergone DBS to those that were only treated with pharmacological intervention, and observed a decrease in the binding of dopamine transporters in the striatum before and after intervention in both groups, suggesting a nigrostriatal neuronal degeneration.

Two further studies $[53,56]$ investigated a potential disease-modifying effect of DBS but did not report any significant results. Ge et al. [53] examined PD-related metabolic covariance patterns (PDRP) after STN-DBS surgery but did not find any significant differences at 12 months of follow-up compared to baseline. Hilker et al. [56], found a significant decrease in the uptake of striatal 18F-dopa in PD subjects, thus revealing a decline in dopaminergic function even in PD subjects that were effectively treated with STN stimulation.

On the other hand, one study [63] observed a correlation between the clinical outcomes, specifically an improvement in tremor and depressive symptoms, along with a decrease in VMAT2 (vesicular monoamine transporter 2) in the striatum, associative striatum, and extra-striatum. Since a decrease in VMAT2 has been associated with increased dopamine [70], the authors proposed that DBS might be related to an increase in dopaminergic activity. However, this study followed up PD patients only once after DBS; thus, the authors were not able to report other relevant results during the subsequent months.

The remaining nine studies $[52,54,55,58-60,61,62,64]$ also investigated several biomarkers but did not report any potential disease-modifying effect. However, two studies [54, 59] examined different biological aspects and reported some interesting findings. One study [54] suggested that 
the improvements induced by DBS in PD symptoms could be due to an interaction between the cerebellum and the putamen, while the second study [59] suggested that pontine glutamine (Glx) and basal ganglia Glutamate (Glu) levels were potentially significant predictors of the efficacy of DBS, indicating a role of glutamatergic neurotransmission in the therapeutic mechanism of DBS.

\section{Discussion}

In this systematic review, we summarized the available evidence on several biological and physiological processes that were associated with DBS in human participants with PD. The main objective was to understand if these processes could be connected to a potential disease-modifying effect.

Only three studies $[28,30,32]$ reported significant results, suggesting a potential disease-modifying effect of DBS. All these studies examined post-mortem brain tissue. Specifically, Mallach et al. [28] reported that DBS might have a role in inhibiting or reversing the decrease in mitochondrial volume, as well as the number of dopaminergic striatal neurons, caused by disease progression. The authors explained that this could be associated with the possible effect of DBS in inhibiting STN, which would subsequently inhibit the glutamate excitotoxicity in the SNpc [28]. Furthermore, the possible long-term consequences of decreased glutamate excitotoxicity would be the loss of calcium-dependent mitochondrial fragmentation, increased mitochondrial volume, and decreased neuronal death [28].

Furthermore, Pienaar et al. [30] suggested an association between STN-DBS and an improvement of microvascular markers. The authors observed a dramatic increase in VEGF, a neurotrophic protein produced by vascular endothelial cells, which may stimulate vasculogenesis and angiogenesis. Therefore, these results indicated that STN-DBS could reverse the extent of vascular pathology in PD by stimulating the survival, proliferation, and migration of vascular endothelial cells. The third study [32] reported an effect of DBS in increasing the cellular plasticity in the brain, suggesting that the effects of surgery might affect a wider area than that directly surrounding the location of the electrode. The authors observed a higher number of SVZ precursor cells (lateral ventricle and third ventricle) in the brains of patients who had undergone DBS compared to those of healthy controls and subjects who did not undergo surgery. The results revealed an increased proliferation of neural precursor cells in the brains of human participants after DBS surgery and electrical stimulation [32]. These results were consistent with those obtained from a study that suggested that STN-DBS might cause a neurotrophic mechanism of neuroprotection by specifically increasing the levels of BDNF [17]. According to this hypothesis, the release of BDNF could be induced by electrical stimulation, thus explaining why high-frequency stimulation in neuronal cultures appeared to cause an increase in the release of BDNF [71]. All these studies were conducted using small sample sizes; thus, despite reporting some significant results, the studies were not strong enough to support a significant effect of DBS in preventing the progression of PD.

Moreover, the study by Wang et al. [33] reported a potential neuroprotective effect of DBS in patients with PD. The authors suggested that tetranectin could be involved in this mechanism by increasing the levels of dopamine and decreasing the accumulation of abnormal proteins. Similarly, a previous study by Wang et al. [72] reported reduced levels of tetranectin in patients with PD compared to $\mathrm{HC}$, with further evidence suggesting a role of this protein in the degradation 
of misfolded proteins [73]. Thus, the observation of higher levels of tetranectin after DBS could suggest the neuroprotective effect of surgery. However, the exact role of this protein in PD has not yet been clarified, and further studies are required.

However, most of the included studies did not support the disease-modifying hypothesis. Particularly, three studies $[29,42,57]$ compared participants that had undergone DBS with those that were only pharmacologically treated and reported no differences between the two groups in terms of disease progression. Only a few studies [29, 56, 57, 63] have investigated the typical pathobiological and pathophysiological hallmarks of PD. As mentioned before, the typical hallmarks of PD are often associated with the aggregation of $\alpha$-synuclein and dopaminergic degeneration in SNpc and other basal ganglia structures [74]. However, most of the included studies examined different biological and physiological biomarkers. Overall, the biological and neurophysiological aspects covered by the included studies were highly heterogeneous. Few studies focused on similar biological and neurophysiological aspects. For example, the included neurophysiological studies adopted the same procedures (such as PET and MER) but examined different neurophysiological aspects and pathways. Similarly, although the four epigenetic studies had the same study design and population, they investigated different epigenetic variables. This heterogeneity in the biological and physiological aspects prevented a direct comparison of studies and limited the reliability of the results in suggesting any significant disease-modifying effect associated with DBS.

A further limitation of the reliability of results in suggesting a potential disease-modifying effect of DBS was that $60.4 \%$ of the studies had a follow-up of $\leq 1$ year, while $67.4 \%$ of the studies observed patients only once after DBS.

Overall, our review reports a significant effect of STN-DBS only during the ON phase, thus suggesting that the effect might be limited and transient. Some of the included neurophysiological studies have reported that the observed metabolic and physiological changes almost completely disappeared during the OFF phase, again indicating the functional and temporary effects of DBS, consistent with the relapse of severe Parkinsonism in the OFF phase of DBS. Moreover, substantial improvements in the UPDRS scores were observed after surgery, only during the ON phase.

However, it is worth highlighting that, although $55.8 \%$ of the studies did not specify the adopted diagnostic criteria, most of them observed late-stage patients with PD about ten years after diagnosis. The recruitment of late-stage patients with PD could be another factor that may partially explain the lack of significant results suggesting a potential disease-modifying effect of DBS. Most of the loss of putaminal denervation occurs within four years after the diagnosis [75]. Thus, assessment of a potential disease-modifying effect in a PD patient in whom the dopaminergic striatal innervation has been lost long back might be inappropriate [76].

Dysfunction and degeneration of the nigrostriatal system begin long before the diagnosis of PD. It has been estimated that, by the time of onset of motor symptoms and subsequent diagnosis, the patients have already lost half of the striatal dopamine content, along with $30 \%$ of the nigral dopamine neurons [75]. A study by Kordower and colleagues [76] demonstrated that patients reached the diagnosis stage when $50 \%$ of the putaminal denervation had already occurred and that this denervation progressed to an approximately $90 \%$ loss within four years after diagnosis. These results suggest that enrolling a population with an advanced stage of the disease might be inappropriate when investigating the potential neuroprotective effects of specific interventions [76]. Several preclinical studies support a potential disease-modifying effect of DBS. Some studies 
on rats have reported that the use of STN-DBS immediately after the administration of 6-hydroxydopamine (6-OHDA) doubled the number of remaining tyrosine hydroxylase immuno-reactive neurons in the SNpc [77], and the activation of STN-DBS at 1 or 2 weeks after the administration of 6-OHDA protected the remaining SNpc neurons from further degeneration [78, 79]. Similar results were observed in primate models of PD using STN-DBS just before or six days after the administration of 1-methyl-4-phenyl-1,2,3,6-tetrahydropyridine (MPTP) [16].

These findings suggest that to explore the potential disease-modifying effects of DBS, studies should enroll patients with milder symptoms and whose neural circuitry and physiology may still respond to DBS. Based on these criteria, a study [80] reported that DBS was superior to medical therapy alone during early-stage PD, before the appearance of severely debilitating motor complications, suggesting that neurostimulation may be a potential therapy for patients in an early stage of the disease. It must be highlighted that DBS is expensive compared to conventional medical therapy, which, apart from being a non-invasive intervention, facilitates an excellent control of symptoms [81]. However, available evidence on the long-term benefits of DBS is insufficient to support the initiation of DBS at an early stage of the disease. Moreover, despite significant technological progress, stereotactic surgery still has a combined risk of permanent morbidity or mortality of 1-3\%, depending on the surgical center and caseload volume [81]. However, as observed in some of the included articles, DBS has been shown to cause substantial improvements in the health-related quality of life, as well as maintain the improvements in symptoms [82]. Moreover, considering that the risks related to surgery are lower in younger subjects with lower brain atrophy and fewer comorbidities, as well as the fact that younger patients have been reported to benefit the most from the intervention [83], DBS might be considered as a feasible treatment option at an earlier stage of the disease.

Due to several limitations of the included literature, the obtained evidence was inconclusive in identifying a potential disease-modifying effect of DBS. Nevertheless, three post-mortem studies have suggested a potential neuroprotective effect of DBS, which justifies further high-quality epidemiological studies enrolling a larger number of subjects to assess if and how this surgical procedure can modify the course of the disease. Specifically, studies should adopt a more suitable design, focusing on the appropriate selection and recruitment of participants based on homogeneous, validated, and standardized diagnostic criteria, as well as adopting an adequate duration of follow-up, including a sufficient number of observations.

Currently available preclinical literature suggests that STN-DBS could mediate neuroprotection at an early stage of the disease. Based on this premise, carrying out further prospective clinical trials examining the course of PD in early-stage patients could be useful.

Further research should focus on expanding our understanding of the biological and physiological changes in the brain caused by either disease progression or chronic electrical stimulation. Current research on the biological and neurophysiological aspects involved in DBS is still widely heterogeneous. Therefore, it could be crucial to reach a consensus on the most appropriate and reliable biomarkers to assess the efficacy of DBS.

Since PD is a highly complex disease, it is unlikely that a single biomarker will be sufficient. Rather, a panel of established and standardized biomarkers covering a range of metabolic and physiologic processes, including genetic, neuroimaging, and metabolic markers, would be helpful.

We believe that such a result could be useful in supporting neurosurgeons and neurologists in assessing the efficacy of DBS, both in clinical practice and in a research setting. 


\section{Conclusions}

This paper aimed at systematically reviewing the biological and physiological effects of DBS in human patients with PD, focusing on its potential disease-modifying features. The reviewed studies investigated a set of widely heterogeneous and complex biological and neurophysiological aspects, and only a few studies specifically investigated the potential neuroprotective and disease-modifying effects of DBS. Most of the included studies did not support the disease-modifying hypothesis, showing transient biological and neurophysiological effects. All the included studies had some methodological limitations, mostly related to study design and patient recruitment. We believe that identifying a panel of common biomarkers and criteria might be crucial to characterize the features of PD better and assess the potential of DBS to modify the course of the disease.

\section{Author Contributions}

F.S.: data collection and analysis, writing of the manuscript. G.R.: data collection, drafting of the manuscript. L.T.: data collection, drafting of the manuscript. G.S.: data collection. F.T.: data collection. G.R.: data collection. E.L.: methodology, drafting of the manuscript. M.C. (Massimo Corbo): revising the manuscript critically for important intellectual content. M.C. (Marco Canevelli): drafting and revising of the manuscript. N.V.: conception of the study, revising of the manuscript. P.P.: data collection, drafting and revising of the manuscript.

\section{Funding}

This work did not receive any specific grant from funding agencies in the public, commercial, or not-for-profit sectors.

\section{Competing Interests}

The authors have declared that no competing interests exist.

\section{References}

1. Kalia LV, Lang AE. Parkinson's disease. Lancet. 2015; 386: 896-912.

2. Jakobs M, Lee DJ, Lozano AM. Modifying the progression of Alzheimer's and Parkinson's disease with deep brain stimulation. Neuropharmacology. 2020; 171: 107860.

3. Dexter DT, Jenner P. Parkinson disease: From pathology to molecular disease mechanisms. Free Radic Biol Med. 2013; 62: 132-144.

4. Benabid AL. Deep brain stimulation for Parkinson's disease. Curr Opin Neurobiol. 2003; 13: 696-706.

5. Bejjani BP, Damier P, Arnulf I, Thivard L, Bonnet AM, Dormont D, et al. Transient acute depression induced by high-frequency deep-brain stimulation. N Engl J Med. 1999; 340: 1476-1480.

6. Kumar R, Lozano AM, Sime E, Halket E, Lang AE. Comparative effects of unilateral and bilateral subthalamic nucleus deep brain stimulation. Neurology. 1999; 53: 561-566.

7. Thobois S, Mertens $P$, Guenot $M$, Hermier $M$, Mollion $H$, Bouvard $M$, et al. Subthalamic 
nucleus stimulation in Parkinson's disease: Clinical evaluation of 18 patients. J Neurol. 2002; 249: 529-534.

8. Berney A, Vingerhoets F, Perrin A, Guex P, Villemure JG, Burkhard PR, et al. Effect on mood of subthalamic DBS for Parkinson's disease: A consecutive series of 24 patients. Neurology. 2002; 59: 1427-1429.

9. St George RJ, Carlson-Kuhta P, Burchiel KJ, Hogarth P, Frank N, Horak FB. The effects of subthalamic and pallidal deep brain stimulation on postural responses in patients with Parkinson disease. J Neurosurg. 2012; 116: 1347-1356.

10. Chastan N, Westby GW, Yelnik J, Bardinet E, Do MC, Agid Y, et al. Effects of nigral stimulation on locomotion and postural stability in patients with Parkinson's disease. Brain. 2009; 132: 172-184.

11. Hashimoto T, Elder CM, Okun MS, Patrick SK, Vitek JL. Stimulation of the subthalamic nucleus changes the firing pattern of pallidal neurons. J Neurosci. 2003; 23: 1916-1923.

12. Kalia SK, Sankar T, Lozano AM. Deep brain stimulation for Parkinson's disease and other movement disorders. Curr Opin Neurol. 2013; 26: 374-380.

13. Dong J, Cui Y, Li S, Le W. Current pharmaceutical treatments and alternative therapies of Parkinson's disease. Curr Neuropharmacol. 2016; 14: 339-355.

14. Limousin P, Pollak P, Benazzouz A, Hoffmann D, Le Bas JF, Broussolle E, et al. Effect of parkinsonian signs and symptoms of bilateral subthalamic nucleus stimulation. Lancet. 1995; 345: 91-95.

15. Ashkan K, Rogers P, Bergman H, Ughratdar I. Insights into the mechanisms of deep brain stimulation. Nat Rev Neurol. 2017; 13: 548-554.

16. Wallace BA, Ashkan K, Heise CE, Foote KD, Torres N, Mitrofanis J, et al. Survival of midbrain dopaminergic cells after lesion or deep brain stimulation of the subthalamic nucleus in MPTP-treated monkeys. Brain. 2007; 130: 2129-2145.

17. Spieles-Engemann AL, Steece-Collier K, Behbehani MM, Collier TJ, Wohlgenant SL, Kemp CJ, et al. Subthalamic nucleus stimulation increases brain derived neurotrophic factor in the nigrostriatal system and primary motor cortex. J Parkinsons Dis. 2011; 1: 123-136.

18. Rodriguez MC, Obeso JA, Olanow CW. Subthalamic nucleus-mediated excitotoxicity in Parkinson's disease: A target for neuroprotection. Ann Neurol. 1998; 44: S175-S188.

19. Tagliati M, Martin C, Alterman R. Lack of motor symptoms progression in Parkinson's disease patients with long-term bilateral subthalamic deep brain stimulation. Int J Neurosci. 2010; 120: 717-723.

20. Higgins JP, Altman DG, Gøtzsche PC, Jüni $P$, Moher D, Oxman AD, et al. The Cochrane Collaboration's tool for assessing risk of bias in randomised trials. BMJ. 2011; 343: d5928.

21. Liberati A, Altman DG, Tetzlaff J, Mulrow C, Gøtzsche PC, loannidis JP, et al. The PRISMA statement for reporting systematic reviews and meta-analyses of studies that evaluate healthcare interventions: Explanation and elaboration. BMJ. 2009; 339: b2700.

22. Constantinescu R, Holmberg B, Rosengren L, Corneliusson O, Johnels B, Zetterberg H. Light subunit of neurofilament triplet protein in the cerebrospinal fluid after subthalamic nucleus stimulation for Parkinson's disease. Acta Neurol Scand. 2011; 124: 206-210.

23. Constantinescu R, Blennow K, Rosengren L, Eriksson B, Gudmundsdottir T, Jansson $Y$, et al. Cerebrospinal fluid protein markers in PD patients after DBS-STN surgery-A retrospective analysis of patients that underwent surgery between 1993 and 2001. Clin Neurol Neurosurg. 
2018; 174: 174-179.

24. Dong W, Qiu C, Gong D, Jiang X, Liu W, Liu W, et al. Proteomics and bioinformatics approaches for the identification of plasma biomarkers to detect Parkinson's disease. Exp Ther Med. 2019; 18: 2833-2842.

25. Guimarães J, Vieira-Coelho MA, Moura E, Afonso J, Rosas MJ, Vaz R, et al. Urinary profile of catecholamines and metabolites in Parkinson patients with deep brain stimulation. Eur J Neurol. 2014; 21: 353-356.

26. Kwiatek-Majkusiak J, Geremek M, Koziorowski D, Tomasiuk R, Szlufik S, Friedman A. Higher serum levels of pro-hepcidin in patients with Parkinson's disease treated with deep brain stimulation. Neurosci Lett. 2018; 684: 205-209.

27. Kwiatek-Majkusiak J, Geremek M, Koziorowski D, Tomasiuk R, Szlufik S, Friedman A. Serum levels of hepcidin and interleukin 6 in Parkinson's disease. Acta Neurobiol Exp. 2020; 80: 297-304.

28. Mallach A, Weinert M, Arthur J, Gveric D, Tierney TS, Alavian KN. Post mortem examination of Parkinson's disease brains suggests decline in mitochondrial biomass, reversed by deep brain stimulation of subthalamic nucleus. FASEB J. 2019; 33: 6957-6961.

29. Pal GD, Ouyang B, Serrano G, Shill HA, Goetz C, Stebbins G, et al. Comparison of neuropathology in Parkinson's disease subjects with and without deep brain stimulation. Mov Disord. 2017; 32: 274-277.

30. Pienaar IS, Lee CH, Elson JL, McGuinness L, Gentleman SM, Kalaria RN, et al. Deep-brain stimulation associates with improved microvascular integrity in the subthalamic nucleus in Parkinson's disease. Neurobiol Dis. 2015; 74: 392-405.

31. Seifried C, Boehncke S, Heinzmann J, Baudrexel S, Weise L, Gasser T, et al. Diurnal variation of hypothalamic function and chronic subthalamic nucleus stimulation in Parkinson's disease. Neuroendocrinology. 2013; 97: 283-290.

32. Vedam-Mai V, Gardner B, Okun MS, Siebzehnrubl FA, Kam M, Aponso P, et al. Increased precursor cell proliferation after deep brain stimulation for Parkinson's disease: A human study. PLoS ONE. 2014; 9: e88770.

33. Wang ES, Yao HB, Chen YH, Wang G, Gao WW, Sun YR, et al. Proteomic analysis of the cerebrospinal fluid of Parkinson's disease patients pre- and post-deep brain stimulation. Cell Physiol Biochem. 2013; 31: 625-637.

34. Soreq L, Bergman H, Goll Y, Greenberg DS, Israel Z, Soreq H. Deep brain stimulation induces rapidly reversible transcript changes in Parkinson's leucocytes. J Cell Mol Med. 2012; 16: 1496-1507.

35. Soreq L, Salomonis N, Bronstein M, Greenberg DS, Israel Z, Bergman H, et al. Small RNA sequencing-microarray analyses in Parkinson leukocytes reveal deep brain stimulation-induced splicing changes that classify brain region transcriptomes. Front $\mathrm{Mol}$ Neurosci. 2013; 6: 10.

36. Soreq L, Bergman $H$, Israel Z, Soreq H. Deep brain stimulation modulates nonsense-mediated RNA decay in Parkinson's patients' leukocytes. BMC Genom. 2013; 14: 478.

37. Soreq L, Guffanti A, Salomonis N, Simchovitz A, Israel Z, Bergman H, et al. Long non-coding RNA and alternative splicing modulations in Parkinson's leukocytes identified by RNA sequencing. PLoS Comput Biol. 2014; 10: e1003517.

38. Airaksinen K, Butorina A, Pekkonen E, Nurminen J, Taulu S, Ahonen A, et al. Somatomotor mu 
rhythm amplitude correlates with rigidity during deep brain stimulation in Parkinsonian patients. Clin Neurophysiol. 2012; 123: 2010-2017.

39. Anidi C, O'Day JJ, Anderson RW, Afzal MF, Syrkin-Nikolau J, Velisar A, et al. Neuromodulation targets pathological not physiological beta bursts during gait in Parkinson's disease. Neurobiol Dis. 2018; 120: 107-117.

40. Däuper J, Peschel T, Schrader C, Kohlmetz C, Joppich G, Nager W, et al. Effects of subthalamic nucleus (STN) stimulation on motor cortex excitability. Neurology. 2002; 59: 700-706.

41. Fraix V, Pollak P, Vercueil L, Benabid AL, Mauguière F. Effects of subthalamic nucleus stimulation on motor cortex excitability in Parkinson's disease. Clin Neurophysiol. 2008; 119: 2513-2518.

42. Giannicola G, Rosa M, Servello D, Menghetti C, Carrabba G, Pacchetti C, et al. Subthalamic local field potentials after seven-year deep brain stimulation in Parkinson's disease. Exp Neurol. 2012; 237: 312-317.

43. Gulberti A, Moll CK, Hamel W, Buhmann C, Koeppen JA, Boelmans K, et al. Predictive timing functions of cortical beta oscillations are impaired in Parkinson's disease and influenced by L-DOPA and deep brain stimulation of the subthalamic nucleus. Neuroimage Clin. 2015; 9: 436-449.

44. Jech R, Růzicka E, Urgosík D, Serranová T, Volfová M, Nováková O, et al. Deep brain stimulation of the subthalamic nucleus affects resting EEG and visual evoked potentials in Parkinson's disease. Clin Neurophysiol. 2006; 117: 1017-1028.

45. Michmizos KP, Frangou $P$, Stathis $P$, Sakas D, Nikita KS. Beta-band frequency peaks inside the subthalamic nucleus as a biomarker for motor improvement after deep brain stimulation in Parkinson's disease. IEEE J Biomed Health Inform. 2015; 19: 174-180.

46. Pierantozzi M, Mazzone P, Bassi A, Rossini PM, Peppe A, Altibrandi MG, et al. The effect of deep brain stimulation on the frontal N30 component of somatosensory evoked potentials in advanced Parkinson's disease patients. Clin Neurophysiol. 1999; 110: 1700-1707.

47. Ray NJ, Jenkinson N, Wang S, Holland, P, Brittain JS, Joint C, et al. T. Local field potential beta activity in the subthalamic nucleus of patients with Parkinson's disease is associated with improvements in bradykinesia after dopamine and deep brain stimulation. Exp Neurol. 2008; 213: 108-113.

48. Rosa M, Giannicola G, Servello D, Marceglia S, Pacchetti C, Porta M, et al. Subthalamic local field beta oscillations during ongoing deep brain stimulation in Parkinson's disease in hyperacute and chronic phases. Neurosignals. 2011; 19: 151-162.

49. Sinclair NC, McDermott HJ, Bulluss KJ, Fallon JB, Perera T, Xu SS, et al. Subthalamic nucleus deep brain stimulation evokes resonant neural activity. Ann Neurol. 2018; 83: 1027-1031.

50. Trager MH, Koop MM, Velisar A, Blumenfeld Z, Nikolau JS, Quinn EJ, et al. Subthalamic beta oscillations are attenuated after withdrawal of chronic high frequency neurostimulation in Parkinson's disease. Neurobiol Dis. 2016; 96: 22-30.

51. Weiss D, Klotz R, Govindan RB, Scholten M, Naros G, Ramos-Murguialday A, et al. Subthalamic stimulation modulates cortical motor network activity and synchronization in Parkinson's disease. Brain. 2015; 138: 679-693.

52. Dong W, Qiu C, Jiang X, Shen B, Zhang L, Liu W, et al. Can the executive control network be used to diagnose Parkinson's disease and as an efficacy indicator of deep brain stimulation? Parkinsons Dis. 2020; 2020: 6348102. 
53. Ge J, Wang M, Lin W, Wu P, Guan Y, Zhang H, et al. Metabolic network as an objective biomarker in monitoring deep brain stimulation for Parkinson's disease: A longitudinal study. EJNMMI Res. 2020; 10: 131.

54. Hanssen H, Steinhardt J, Münchau A, Al-Zubaidi A, Tzvi E, Heldmann M, et al. Cerebello-striatal interaction mediates effects of subthalamic nucleus deep brain stimulation in Parkinson's disease. Parkinsonism Relat Disord. 2019; 67: 99-104.

55. Hilker R, Voges J, Weisenbach S, Kalbe E, Burghaus L, Ghaemi M, et al. Subthalamic nucleus stimulation restores glucose metabolism in associative and limbic cortices and in cerebellum: Evidence from a FDG-PET study in advanced Parkinson's disease. J Cereb Blood Flow Metab. 2004; 24: 7-16.

56. Hilker R, Portman AT, Voges J, Staal MJ, Burghaus L, van Laar T, et al. Disease progression continues in patients with advanced Parkinson's disease and effective subthalamic nucleus stimulation. J Neurol Neurosurg Psychiatry. 2005; 76: 1217-1221.

57. Lokkegaard A, Werdelin LM, Regeur L, Karlsborg M, Jensen SR, Brødsgaard E, et al. Dopamine transporter imaging and the effects of deep brain stimulation in patients with Parkinson's disease. Eur J Nucl Med Mol Imaging. 2007; 34: 508-516.

58. Mubeen AM, Ardekani B, Tagliati M, Alterman R, Dhawan V, Eidelberg D, et al. Global and multi-focal changes in cerebral blood flow during subthalamic nucleus stimulation in Parkinson's disease. J Cereb Blood Flow Metab. 2018; 38: 697-705.

59. O'Gorman Tuura RL, Baumann CR, Baumann-Vogel H. Neurotransmitter activity is linked to outcome following subthalamic deep brain stimulation in Parkinson's disease. Parkinsonism Relat Disord. 2018; 50: 54-60.

60. Palard-Novello X, Drapier S, Bonnet A, Girard A, Robert G, Houvenaghel JF, et al. Deep brain stimulation of the internal globus pallidus does not affect the limbic circuit in patients with Parkinson's disease: A PET study. J Neurol. 2021; 268: 701-706.

61. Péron J, Le Jeune F, Haegelen C, Dondaine T, Drapier D, Sauleau P, et al. Subthalamic nucleus stimulation affects theory of mind network: A pet study in Parkinson's disease. PLoS ONE. 2010; 5: e9919.

62. Sidtis JJ, Tagliati M, Alterman R, Sidtis D, Dhawan V, Eidelberg D. Therapeutic high-frequency stimulation of the subthalamic nucleus in Parkinson's disease produces global increases in cerebral blood flow. J Cereb Blood Flow Metab. 2012; 32: 41-49.

63. Smith GS, Mills KA, Pontone GM, Anderson WS, Perepezko KM, Brasic J, et al. Effect of STN DBS on vesicular monoamine transporter 2 and glucose metabolism in Parkinson's disease. Parkinsonism Relat Disord. 2019; 64: 235-241.

64. Vassal F, Dilly D, Boutet C, Bertholon F, Charier D, Pommier B. White matter tracts involved by deep brain stimulation of the subthalamic nucleus in Parkinson's disease: A connectivity study based on preoperative diffusion tensor imaging tractography. $\mathrm{Br} J$ Neurosurg. 2020; 34: 187-195.

65. Gibb WR, Lees AJ. A comparison of clinical and pathological features of young- and old-onset Parkinson's disease. Neurology. 1988; 38: 1402-1406.

66. Hoehn MM, Yahr MD. Parkinsonism: Onset, progression and mortality. Neurology. 1967; 17: 427-442.

67. Postuma RB, Berg D, Stern M, Poewe W, Olanow CW, Oertel W, et al. MDS clinical diagnostic criteria for Parkinson's disease. Mov Disord. 2015; 30: 1591-1601. 
68. Nagatsu T, Sawada M. Inflammatory process in Parkinson's disease: Role for cytokines. Curr Pharm Des. 2005; 11: 999-1016.

69. Thompson JA, Lanctin D, Ince NF, Abosch A. Clinical implications of local field potentials for understanding and treating movement disorders. Stereotact Funct Neurosurg. 2014; 92: 251-263.

70. Boileau I, Rusjan P, Houle S, Wilkins D, Tong J, Selby P, et al. Increased vesicular monoamine transporter binding during early abstinence in human methamphetamine users: Is VMAT2 a stable dopamine neuron biomarker? J Neurosci. 2008; 28: 9850-9856.

71. Hartmann M, Heumann R, Lessmann V. Synaptic secretion of BDNF after high-frequency stimulation of glutamatergic synapses. EMBO J. 2001; 20: 5887-5897.

72. Wang ES, Sun Y, Guo JG, Gao X, Hu JW, Zhou L, et al. Tetranectin and apolipoprotein A-I in cerebrospinal fluid as potential biomarkers for Parkinson's disease. Acta Neurol Scand. 2010; 122: 350-359.

73. Dietzmann K, von Bossanyi $P$, Krause D, Wittig $H$, Mawrin C, Kirches E. Expression of the plasminogen activator system and the inhibitors PAI-1 and PAI-2 in posttraumatic lesions of the CNS and brain injuries following dramatic circulatory arrests: An immunohistochemical study. Pathol Res Pract. 2000; 196: 15-21.

74. Mugge L, Krafcik B, Pontasch G, Alnemari A, Neimat J, Gaudin D. A review of biomarkers use in Parkinson with deep brain stimulation: A successful past promising a bright future. World Neurosurg. 2019; 123: 197-207.

75. Cheng HC, Ulane CM, Burke RE. Clinical progression in Parkinson disease and the neurobiology of axons. Ann Neurol. 2010; 67: 715-725.

76. Kordower JH, Olanow CW, Dodiya HB, Chu Y, Beach TG, Adler CH, et al. Disease duration and the integrity of the nigrostriatal system in Parkinson's disease. Brain. 2013; 136: 2419-2431.

77. Maesawa S, Kaneoke Y, Kajita Y, Usui N, Misawa N, Nakayama A, et al. Long-term stimulation of the subthalamic nucleus in hemiparkinsonian rats: Neuroprotection of dopaminergic neurons. J Neurosurg. 2004; 100: 679-687.

78. Temel Y, Visser-Vandewalle V, Kaplan S, Kozan R, Daemen MA, Blokland A, et al. Protection of nigral cell death by bilateral subthalamic nucleus stimulation. Brain Res. 2006; 1120: 100-105.

79. Harnack D, Meissner W, Jira JA, Winter C, Morgenstern R, Kupsch A. Placebo-controlled chronic high-frequency stimulation of the subthalamic nucleus preserves dopaminergic nigral neurons in a rat model of progressive Parkinsonism. Exp Neurol 2008; 210: 257-260.

80. Schuepbach WM, Rau J, Knudsen K, Volkmann J, Krack P, Timmermann L, et al. Neurostimulation for Parkinson's disease with early motor complications. N Engl J Med. 2013; 368: 610-622.

81. Limousin P, Foltynie T. Long-term outcomes of deep brain stimulation in Parkinson disease. Nat Rev Neurol. 2019; 15: 234-242.

82. Malek N. Deep brain stimulation in Parkinson's disease. Neurol India. 2019; 67: 968-978.

83. Williams A, Gill S, Varma T, Jenkinson C, Quinn N, Mitchell R, et al. Deep brain stimulation plus best medical therapy versus best medical therapy alone for advanced Parkinson's disease (PD SURG trial): A randomised, open-label trial. Lancet Neurol. 2010; 9: 581-591. 


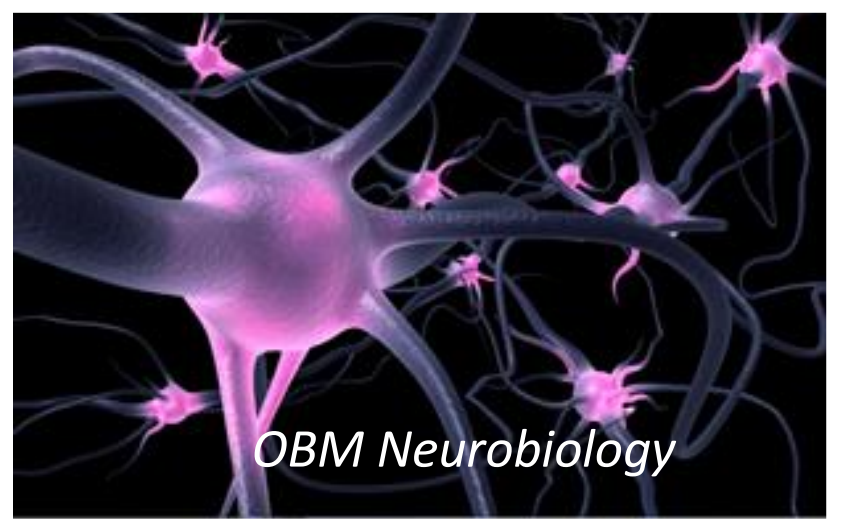

Enjoy OBM Neurobiology by:

1. Submitting a manuscript

2. Joining volunteer reviewer bank

3. Joining Editorial Board

4. Guest editing a special issue

For more details, please visit:

http://www.lidsen.com/journals/neurobiology 